\title{
Resurrection of a functional phosphatidylinositol transfer protein from a pseudo-Sec14 scaffold by directed evolution
}

\author{
Gabriel Schaafa,b, Marek Dynowskib,c, Carl J. Mousley ${ }^{a}$, Sweety D. Shah ${ }^{a}$, Peihua Yuan ${ }^{a}$, \\ Eva M. Winklbauerb, Marília K. F. de Campos ${ }^{b}$, Kyle Trettin ${ }^{a}$, Mary-Chely Quinones ${ }^{d}$, \\ Tatyana I. Smirnovad, Lora L. Yanagisawae, Eric A. Ortlundf, and Vytas A. Bankaitis ${ }^{a}$ \\ aDepartment of Cell and Developmental Biology, Lineberger Comprehensive Cancer Center, University of North \\ Carolina School of Medicine, Chapel Hill, NC 27599; ${ }^{\circ}$ Center for Plant Molecular Biology, Plant Physiology, Universität \\ Tübingen, 72076 Tübingen, Germany; ${ }^{c}$ Rechenzentrum, Universität Freiburg, 79104 Freiburg, Germany; ${ }^{d}$ Department \\ of Chemistry, North Carolina State University, Raleigh, NC 27695; eDepartment of Cell Biology, University of Alabama- \\ Birmingham School of Medicine, Birmingham, AL 35294; fDepartment of Biochemistry, Emory University School of \\ Medicine, Atlanta, GA 30322
}

\begin{abstract}
Sec14-superfamily proteins integrate the lipid metabolome with phosphoinositide synthesis and signaling via primed presentation of phosphatidylinositol (Ptdlns) to Ptdlns kinases. Sec14 action as a Ptdlns-presentation scaffold requires heterotypic exchange of phosphatidylcholine (PtdCho) for Ptdlns, or vice versa, in a poorly understood progression of regulated conformational transitions. We identify mutations that confer Sec14-like activities to a functionally inert pseudo-Sec14 (Sfh1), which seemingly conserves all of the structural requirements for Sec14 function. Unexpectedly, the "activation" phenotype results from alteration of residues conserved between Sfh1 and Sec14. Using biochemical and biophysical, structural, and computational approaches, we find the activation mechanism reconfigures atomic interactions between amino acid side chains and internal water in an unusual hydrophilic microenvironment within the hydrophobic Sfh1 ligand-binding cavity. These altered dynamics reconstitute a functional "gating module" that propagates conformational energy from within the hydrophobic pocket to the helical unit that gates pocket access. The net effect is enhanced rates of phospholipid-cycling into and out of the Sfh1* hydrophobic pocket. Taken together, the directed evolution approach reveals an unexpectedly flexible functional engineering of a Sec14-like Ptdlns transfer protein-an engineering invisible to standard bioinformatic, crystallographic, and rational mutagenesis approaches.
\end{abstract}

\author{
Monitoring Editor \\ Reid Gilmore \\ University of Massachusetts
}

Received: Nov 18, 2010

Revised: Jan 6, 2011

Accepted: Jan 10, 2011

\footnotetext{
This article was published online ahead of print in MBoC in Press (http://www .molbiolcell.org/cgi/doi/10.1091/mbc.E10-11-0903) on January 19, 2011. The authors declare no conflict of interest.

Address correspondence to: Vytas A. Bankaitis (vytas@med.unc.edu) or Gabriel Schaaf (gabriel.schaaf@zmbp.uni-tuebingen.de).

Abbreviations used: CPY, carboxypeptidase Y; CW, continuous wave; EPR, electron paramagnetic resonance; $\mathrm{MD}$, molecular dynamics; PIP, phosphoinositide; PITP, Ptdlns-transfer protein; PL, phospholipid; PLD, phospholipase D; PtdCho, phosphatidylcholine; PtdEtn, phosphatidylethanolamine; Ptdlns, phosphatidylinositol; rmsd, root mean square deviation; rmsf, root mean square fluctuation; TGN, trans-Golgi network; YPD, yeast peptone dextrose.

(C) 2011 Schaaf et al. This article is distributed by The American Society for Cell Biology under license from the author(s). Two months after publication it is available to the public under an Attribution-Noncommercial-Share Alike 3.0 Unported Creative Commons License (http://creativecommons.org/licenses/by-nc-sa/3.0). "ASCB ${ }^{\circledR}$," "The American Society for Cell Biology ${ }^{\circledR}$," and "Molecular Biology of the Cell ${ }^{\circledR} "$ are registered trademarks of The American Society of Cell Biology.
}

\section{INTRODUCTION}

Phosphorylated derivatives of phosphatidylinositol (Ptdlns), or phosphoinositides (PIPs), are elementary components of membrane-associated signaling systems in eukaryotes (Majerus, 1997; Fruman et al., 1998; McLaughlin and Murray, 2005; lle et al., 2006; Strahl and Thorner, 2007). The remarkable diversity of PIP signaling is established by two major factors. First, enzymes that produce PIPs are differentially localized within cells, thereby facilitating compartmentspecific enrichment of individual PIP species. Second, PIP chemical heterogeneities are interpreted by proteins harboring headgroupbinding domains with appropriate positional specificities. The diversity in PIP signaling promoted by chemical heterogeneity is subject to further amplification by combinatorial mechanisms that couple biological recognition of a PIP-dependent event to a second molecular 
interaction (Balla, 2005; Lemmon, 2008). Such coincidence detection is termed a "back-loaded" strategy on the principle that diversification occurs once the PIP has been generated (Bankaitis et al., 2010).

Recent studies demonstrate that production of an individual PIP species by a specific Ptdlns kinase evokes diverse biological outcomes in a single cell (Routt et al., 2005). Those findings are difficult to explain by back-loaded mechanisms alone. Rather, front-loaded strategies also contribute to diversification of PIP signaling outcomes (Schaaf et al., 2008; Bankaitis et al., 2010). The principle that underlies front-loaded mechanisms highlights an intrinsic biological inadequacy of Ptdlns kinases (particularly Ptdlns 4-OH kinases), which are insufficient interfacial enzymes when confronted with the dual issues of 1) substrate Ptdlns presented in liposomal contexts and 2) competing activities that degrade PIPs. Productive action of these enzymes is subject to what we term "instructive" activation (Bankaitis et al., 2010). Sec14-like Ptdlns-transfer proteins (PITPs) are the instructive components in front-loaded regulation of Ptdlns 4-OH kinase activities in yeast by virtue of their roles as primeable "Ptdlns-presentation" modules. For Sec14, effective presentation requires heterotypic Ptdlns/phosphatidylcholine (PtdCho) exchange (Schaaf et al., 2008; Bankaitis et al., 2010). The Sec14-protein superfamily is diverse, and a Ptdlns-binding "bar code" is recognized in nearly all members of the superfamily. PtdCho-binding signatures are not broadly conserved, however, forecasting that lipids other than PtdCho are sensed by Sec14-like proteins and that metabolism of these lipids might also be coupled to PIP synthesis by Sec14-like proteins. Thus the Sec14 superfamily might link diverse territories of the lipid metabolome with instructed PIP signaling (Schaaf et al., 2008; Bankaitis et al., 2010). This interface is of interest given the causal links between Sec14-like protein deficiencies and human disease (Ouachi et al., 1995; Benomar et al., 2002; Bomar et al., 2003; Meier et al., 2003; D'Angelo et al., 2006).

Understanding the mechanics of how Sec14-like PITPs execute phospholipid (PL) exchange is of central importance given heterotypic PL-exchange reactions lie at the heart of the mechanisms by which these proteins integrate lipid metabolism with PIP signaling (Bankaitis et al.,1990, 2010; Cleves et al., 1991). To interrogate the functional engineering of Sec14-like proteins, we used a directed evolution approach to resurrect Sec14-like activities in a protein (Sfh1) that substantially lacks them. We find single residue substitutions, involving conserved residues, are sufficient to endow the Sfh1 "pseudo-Sec14" with Sec14-like activities in yeast (Sfh1*). These properties are associated with enhanced abilities of Sfh 1 * to cycle PL into and out of the protein interior in vitro and stimulate Ptdlns kinase activities in vivo. The resurrected Sec14-like activities result from altered atomic interactions involving an unusual hydrophilic microenvironment within the hydrophobic Sfh1 ligand-binding cavity. These altered dynamics facilitate conformational transitions in core structural elements that control PL exchange from the Sfh 1 hydrophobic pocket. Taken together, these studies provide new mechanistic insights into the functional design of a Sec14-like PITP.

\section{RESULTS}

\section{Isolation of SFH1* alleles that endow Sfh1 with Sec14-like activities}

Enhanced expression of Sfh 1, the protein most closely related to the essential yeast Sec14, fails to rescue phenotypes associated with Sec14 defects. Moreover, Sfh1 does not catalyze robust Ptdlns- or PtdCho-transfer activity in vitro (Li et al., 2000). Yet Sfh1 shares 64\% primary sequence identity with Sec14 and conserves all of the obvi- ous Sec14 structural motifs critical for Ptdlns and PtdCho binding (Schaaf et al., 2008). These findings suggest either that Sfh1 is a pseudo-Sec14 that is unable to effectively undergo the conformational dynamics that accompany PL transfer reactions in Sec14 or that these transitions fundamentally differ from those of Sec14. Our description of Sfh1 as a pseudo-Sec14 holds no implied evolutionary connotation. This convenient descriptor simply reflects that Sfh1, while sharing high primary sequence similarity with Sec14, is not functionally an active Sec14.

To better understand the mechanics of how Sec14-like PITPs function at the single molecule level, we sought to resurrect Sec14-like activities in Sfh1. To that end, missense mutations were incorporated into the SFH1 gene by error-prone PCR and in vivo gap repair, and reconstituted plasmids driving expression of mutagenized SFH1 genes were introduced into a sec14-1 $1^{\text {ts }}$ ura3-52 yeast strain. Transformants were coselected for growth at $37^{\circ} \mathrm{C}$ and uracil prototrophy. From an estimated $1 \times 10^{6}$ potential Ura ${ }^{+}$transformants, $486 \mathrm{Ura}^{+} \mathrm{Ts}^{+}$ transformants were recovered $\left(-0.05 \%\right.$ of potential $\mathrm{Ura}^{+}$transformants). Of these, 100 were saved for detailed analysis. In all cases, the $\mathrm{Ts}^{+}$growth phenotype was plasmid linked on the basis of two criteria. First, plasmid loss induced by URA3 counterselection with 5-fluoroorotic acid was accompanied by loss of the $\mathrm{Ts}^{+}$phenotype. Reciprocally, SFH1 plasmids were recovered from each of the parental Ura ${ }^{+} \mathrm{Ts}^{+}$ transformants and reintroduced into naïve sec14-1 ${ }^{\text {ts }}$ mutants by selection for $\mathrm{Ura}^{+}$. In each case, the $\mathrm{Ts}^{+}$phenotype was coinherited with plasmid. We refer to these SFH1 activation alleles as SFH1*.

\section{Single substitutions activate Sec14-like functions in Sfh1}

Of the $100 \mathrm{SFH1}$ * isolates, complete nucleotide sequences were determined for 40 clones. These analyses demonstrated the SFH1* isolates contained an average of 4.4 nucleotide and 3.1 amino acid substitutions per gene and gene product, respectively (Figure 1A, Supplemental Figure S1). Two hot spots for SFH1* activation alleles were identified. One hot spot includes Sfh 1 residues $Y_{109}$ and $Y_{113}$, while the other highlights the sequence between residues $I_{131}$ and $T_{141}$ (Figure 1A; Supplemental Figure S1). The $Y_{109} / Y_{113}$ hotspot involves an enigmatic hydrophilic patch on the floor of the Sfh1 hydrophobic cavity (Smirnova et al., 2006; Schaaf et al., 2008). This patch is configured in close proximity to the sn-2 acyl chain of bound PtdCho (Figure 1B) and Ptdlns (Figure 1C). The hydrophilic patch surface is organized by residues $Y_{109}, Q_{111}, Y_{124}$, and $E_{126}$ and involves several coordinated $\mathrm{H}_{2} \mathrm{O}$ molecules, one of which resides in close proximity to $Y_{113}$. The $\mathrm{Q}_{204} \mathrm{R}$ substitution was also identified in the $\mathrm{SFH1}{ }^{*}$ screen. As Sfh $1^{\text {O204R }}$ is unique among the Sfh1* , in that it is far removed from the hydrophilic patch, details of its characterization will be presented elsewhere.

Incorporation of individual $\mathrm{Y}_{109} \mathrm{C}, \mathrm{Y}_{109} \mathrm{H}, \mathrm{Y}_{109} \mathrm{~F}$, and $\mathrm{Y}_{113} \mathrm{C}$ substitutions into an otherwise wild-type $\mathrm{Sfh} 1$ protein demonstrated sufficiency of these substitutions for Sfh ${ }^{*}$ (Figure 2A). Because several substitutions at residue $Y_{109}$ yielded $S f h 1^{*}$, the analyses were extended by determining whether the $Y_{109} A$ substitution (a side chain deletion) had the same effect. Indeed, $Y_{109} A$ conferred Sfh $1{ }^{*}$ properties to an otherwise unadulterated Sfh1 (Figure 2A).

Because the $\mathrm{Y}_{109} \rightarrow \mathrm{F}, \mathrm{C}, \mathrm{H}, \mathrm{A}$, and $\mathrm{Y}_{113} \rightarrow \mathrm{C}$ all represent Sfh1* substitutions, and all affect the enigmatic hydrophilic patch, we tested whether Sfh1E126A and Sfh10111A (i.e., substitutions not encountered in the original activation screen) similarly manifest Sfh 1 * properties when expressed from centromeric and episomal plasmids in sec14-1 $1^{\text {ts }}$ and $\sec 14 \Delta$ shuffle strains. Both Sfh $1^{\mathrm{E} 126 \mathrm{~A}}$ and Sfh 1 ${ }^{\mathrm{Q} 111 \mathrm{~A}}$ scored as Sfh1*. Sfh1 $1^{\mathrm{E} 126 \mathrm{~A}}$ was particularly potent in this regard (Figure 2A). Sfh $1^{\text {Q111A }}$ was less so-primarily due to reduced stability of this protein (discussed later in this article). 
$109 \quad 113$

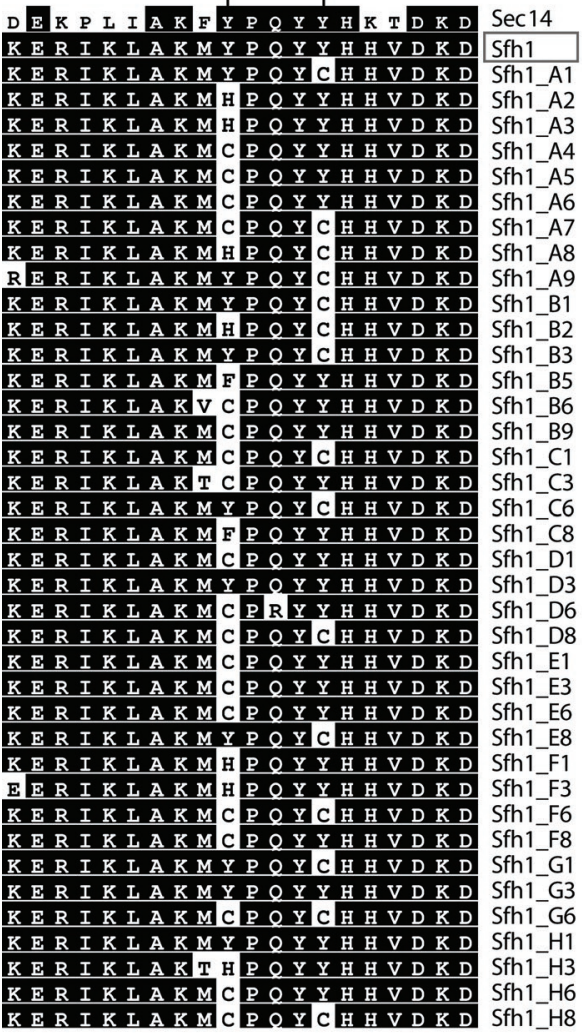

B
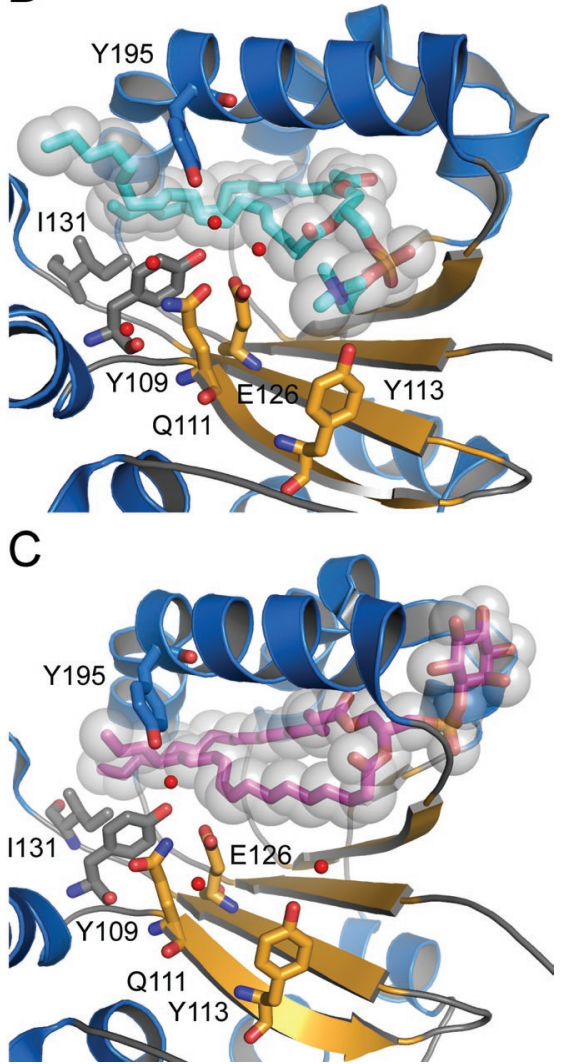

FIGURE 1: SFH1* activation alleles. (A) Protein alignment of Sfh1* mutants obtained in the primary directed evolution screen. Depicted are amino acid residues 100-119. The sequences of Sec14 and the Sfh1 wild-type protein are presented for comparison. (B and C) Ribbon diagrams illustrating the hydrophilic patch at the floor of the hydrophobic PL-binding pocket. Sfh1 residues that are substituted by missense mutations in Sfh1* are shown as sticks. Water molecules are presented as red spheres. PtdCho is rendered in cyan as sticks and transparent spheres in the Sfh1-PtdCho structure (pdb 3B7Q, B), while Ptdlns is rendered in magenta as sticks and transparent spheres in the Sfh1-Ptdlns structure (pdb 3B7N, C).

\section{Characterization of Sec14-like activities in Sfh1* proteins}

Expression of any one of these SFH1* gene products rescued robust growth of both a sec14-1 ${ }^{\text {ts }}$ strain and its isogenic phospholipase D (PLD)-deficient derivative (sec14-1 ${ }^{\text {ts }}$ spo14 $\Delta$ ) at the restrictive temperature of $37^{\circ} \mathrm{C}$ (Figure 2A). The ability of SFH1* expression to effect phenotypic rescue of $\sec 14-1^{\text {ts }}$ growth defects in the spo $14 \Delta$ genetic background speaks to potency of the activation phenotype as PLD deficiency strongly exacerbates sec14-1 ts $_{\text {t }}$ associated growth phenotypes. By comparison, SFH1 expression fails to rescue sec $14-1^{\text {ts }}$ growth defects at $37^{\circ} \mathrm{C}$ - even after prolonged incubation (Figure 2A). The potencies of SFH1* alleles were also apparent in plasmid shuffle assays that score their ability to restore viability to sec14 $\Delta$ strains (Phillips et al., 1999; see Materials and Methods). Expression of SFH1* from low-copy plasmids failed to support shuffle of YEp(SEC14), even when SFH1* gene products with two independent activation substitutions were ex-

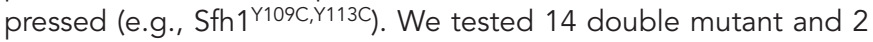
triple mutant combinations in this regard, and in no case did we observe intragenic synergy between $\mathrm{SFH1}^{*}$ mutations (unpublished data). While SFH1 expression failed to support plasmid shuffle under any condition, shuffle of the YEp(SEC14) plasmid was observed when SFH1* expression was driven under control of the powerful plasma membrane ATPase (PMA1) promoter from episomal vectors (Figure 2A).
SFH1* protein expression from low-copy plasmids supports protein accumulation to levels that do not exceed those of endogenous Sec14 (Supplemental Figure S2). Moreover, SFH1 and $\mathrm{SFH}^{*}$ gene products generally accumulated to comparable steady state levels, demonstrating the SFH1* phenotype is not a trivial result of increased protein expression or stability. Exceptions included Sfh $1^{\text {Y109A }}$ and Sfh10111A, both of which exhibited reduced protein stability at $37^{\circ} \mathrm{C}$ relative to Sfh 1 (Supplemental Figure S2). This property is reflected in the modest Sfh 1* phenotypes associated with Sfh $1{ }^{\text {Q111A }}$. However, expression of Sfh $1{ }^{\text {Q111A }}$ rescues growth of sec14- $1^{\text {ts }}$ yeast at $35^{\circ} \mathrm{C}$ (unpublished data) and rescues sec14 14 lethality when expressed from episomal expression plasmids (Figure 2A). The observation that $Y_{109} A$ effects a strong Sfh 1* phenotype, despite reduced expression levels, testifies to its potency.

\section{Sfh1* expression and trans-Golgi network (TGN)/endosomal membrane trafficking}

Phenotypic rescue of Sec14 insufficiencies translates to enhanced ability of Sfh 1 * to execute Sec14-like functions in protein trafficking through the TGN/endosomal system. The accumulation of cytoplasmic toroid structures that represent defective cargoladen TGN/endosomal compartments was readily apparent in thin-section electron micrographs of sec14-1's mutants incubated at restrictive temperatures, and Sfh1 expression failed to rescue this aberrant morphology (Figure 2B). By contrast, this morphological phenotype was alleviated in sec14-1 $1^{\text {ts }}$ mutants by Sfh $1^{\text {Y109A }}$, Sfh $1^{\mathrm{Y} 113 \mathrm{C}}$, or Sfh1 ${ }^{\mathrm{E} 126 \mathrm{~A}}$ expression. Moreover, introduction of the $T_{238} D$ or $S_{175} l, T_{177} l$ missense substitutions (which specifically compromise Ptdlns and PtdCho binding, respectively; discussed later in this article) functionally ablated the Sec14-like functions of these Sfh 1 * proteins.

Pulse-radiolabeling experiments demonstrate that transit of carboxypeptidase $\mathrm{Y}$ (CPY) through the yeast secretory pathway to the vacuole is restored in Sec14-deficient cells by Sfh $1{ }^{*}$ (Figure 2C). In a sec 14- $1^{\text {ts }}$ strain ectopically expressing Sfh 1 , pulse-radiolabeled CPY was recovered in approximately equimolar fractions as TGN/endosomal $\mathrm{p} 2-\mathrm{CPY}$ and mature vacuolar $\mathrm{mCPY}$ forms after a 25-min chase at $37^{\circ} \mathrm{C}$. The persistence of $\mathrm{p} 2-\mathrm{CPY}$ postchase reports a trafficking defect from TGN/endosomes to the vacuole. Cells reconstituted with physiological levels of Sec14 expression presented a single radiolabeled CPY species, the $\mathrm{mCPY}$, under the same experimental conditions. Even modest Sfh1* expression was sufficient to yield CPY profiles that largely recapitulated those recorded for the Sec14-proficient condition. While a persistent p2-CPY fraction remained detectable in the face of Sfh ${ }^{*}$ expression, this fraction was reduced $\sim$ fivefold relative to that recorded in the face of ectopic Sfh1 expression (Figure 2C). The persistence of p2-CPY under conditions of low-copy plasmid-driven SFH1* expression indicates the cognate gene products remain suboptimal Sec14 surrogates. 


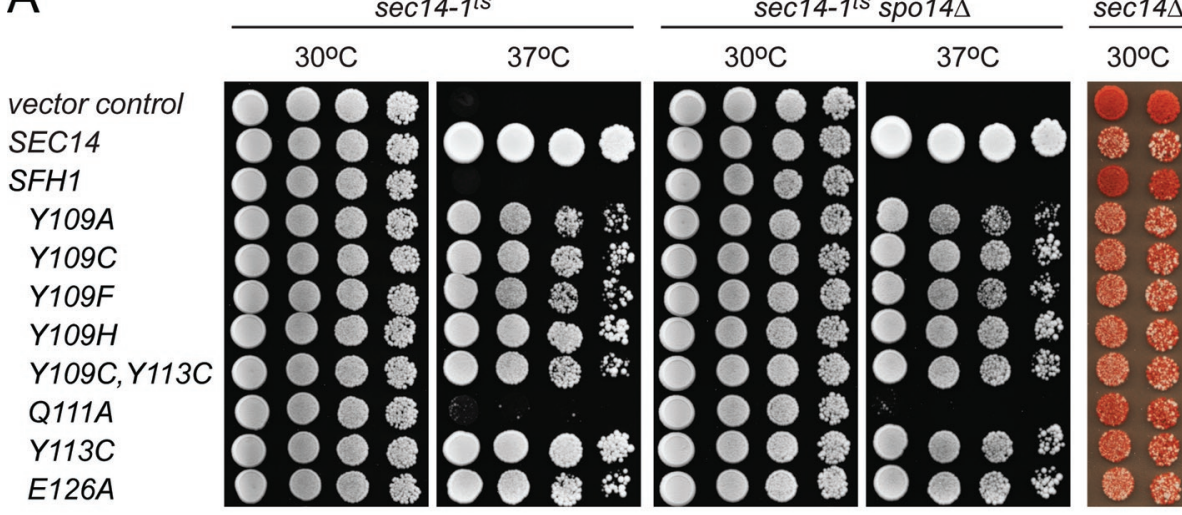

B

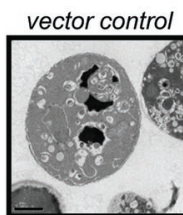

E126A

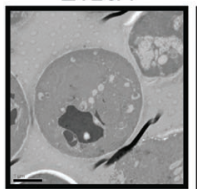

E126A T238D

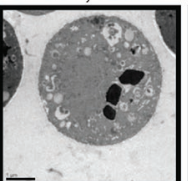

SEC14

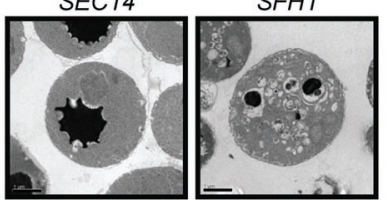

E126A,S175I,T177I

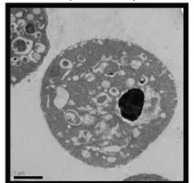

C

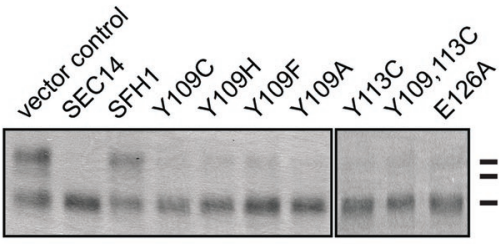

FIGURE 2: Functional characterization of Sfh1* proteins. (A) Left and middle, isogenic sec14-1ts and sec14- $1^{\text {ts }}$ spo $14 \Delta$ yeast strains (as indicated on top) transformed with YCp(URA3) plasmids carrying either $\mathrm{SFH} 1$ or the designated $\mathrm{SFH} 1^{*}$ alleles were spotted in eightfold serial dilutions onto yeast peptone dextrose (YPD) agar plates. Rescue at the restrictive temperature $\left(37^{\circ} \mathrm{C}\right)$ reports Sec14-like activity. YCp(URA3) and YCp(SEC14, URA3) plasmids served as negative and positive controls, respectively. Right, an ade2 ade $3 \sec 14 \Delta$ yeast strain carrying a parental YEp(SEC14, LEU2, ADE3) plasmid (strain CTY558; see Materials and Methods) was transformed with high-copy YEp(URA3) plasmids harboring SFH1 or the designated SFH1* alleles and dilution spotted onto YPD agar plates. Segregation with appearance of white colonies that acquire leucine and histidine auxotrophies reports loss of parental YEp(SEC14, LEU2, ADE3) and hence functionality of the mutant SFH1* product. YEp(URA3) alone and YEp(SEC14, URA3) plasmids served as negative and positive controls, respectively. (B) Thin-section electron microscopy. A sec14-1 $1^{\text {ts }}$ yeast strain (CTY1-1A) expressing the indicated gene (or allele) from a YCp vector was cultured in YPD medium at $30^{\circ} \mathrm{C}$ and subsequently shifted to $37^{\circ} \mathrm{C}$ for $2 \mathrm{~h}$. Cells were fixed, embedded in Spurr's resin, stained with uranyl acetate, and imaged by transmission electron microscopy. Representative images are shown (bar $=1 \mu \mathrm{m})$. (C) A sec $14-1^{\text {ts }}$ yeast strain (CTY1-1A) carrying the indicated YCp expression plasmids was shifted to $37^{\circ} \mathrm{C}$ for $2 \mathrm{~h}$ and radiolabeled with ${ }^{35} \mathrm{~S}$ ]amino acids for $35 \mathrm{~min}$ followed by a 25 -min chase. Immunoprecipitated CPY forms were separated by SDS-PAGE and visualized by autoradiography. Core glycosylated p1 CPY (diagnosing ER and early Golgi pools), TGN p2 CPY, and vacuolar mCPY are identified at right.

FM4-64 tracer experiments similarly demonstrated that Sfh ${ }^{*}$ are superior to Sfh1 in potentiating membrane flow through a Sec14deficient endosomal system. Whereas Sec14-deficient cells internalize the lipophilic dye efficiently, these accumulate FM4-64 in punctate endosomal compartments and fail to chase the tracer into the vacuole (Figure 3). Reconstitution of Sec14-deficient cells with Sec14 rescued these defects. Significant delivery of FM4-64 to vacuolar compartments was already apparent by $7.5 \mathrm{~min}$ of chase, and the endosomal FM4-64 pool quantitatively trafficked to vacuoles during the 30-min chase (Figure 3). Sfh1 expression did not rescue FM4-64 delivery from endosomal compartments to the vacuole, but Sf-

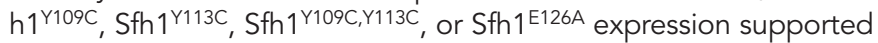

$=\mathrm{p}_{1} \mathrm{CPY}$ - $\mathrm{p} 1 \mathrm{CPY}$ an obvious (yet partial) improvement in dye trafficking from Sec14-deficient endosomes to the vacuole (Figure 3 ).

\section{Sfh1* mutations increase Sfh1-}

\section{mediated stimulation of PIP synthesis}

Sfh1* competence in stimulating PIP production in vivo was quantified by reconstituting SFH1* expression from low-copy vectors in a sec14 14 cki "bypass Sec14" yeast strain (see Materials and Methods). This strain exhibits low basal PIP levels as a consequence of sec14 nullizygosity (Phillips et al., 1999; Routt et al., 2005; Schaaf et al., 2008). Reconstitution of Sec14 expression elevates Ptdlns-3-P, Ptdlns-4-P, and Ptdlns$4,5-\mathrm{P}_{2}$ levels by $\sim$ twofold relative to the parental negative control (Figure 4A). While Sfh1 expression was ineffectual in this regard, even modest expression of Sfh 1 Y109A, Sfh1 ${ }^{\mathrm{Y} 113 \mathrm{C}}$, or Sfh1 ${ }^{\mathrm{E} 126 \mathrm{~A}}$ elevated PIP levels significantly (Figure 4A).

Similar conclusions were drawn from experiments in which the influences of Sfh 1 * proteins and Sfh1 were compared in the isolated context of Stt4 Ptdlns 4-OH kinase activity. To measure Sfh $1{ }^{*}$-mediated potentiation of specific yeast Ptdlns 4-OH kinase activity in a native intracellular environment, $\left[{ }^{3} \mathrm{H}\right]$ Ins radiolabeling experiments were performed in sec14-1ts $\operatorname{sac} 1$ yeast strains expressing the Sfh 1 proteins of interest. The sec14- $1^{\text {ts }}$ sac1 double mutants are defective in Sac1 PIP phosphatase activity and exhibit a dramatic and selective expansion of the Ptdlns-4-P pool generated by the Stt4 Ptdlns 4-OH kinase (Guo et al., 1999; Rivas et al., 1999; Nemoto et al., 2000; Foti et al., 2001). Reconstitution of Sfh $1^{\text {Y109A }, ~ S f-~}$ $\mathrm{h} 1^{\mathrm{Y} 113 \mathrm{C}}$, or Sfh $1^{\mathrm{E} 126 \mathrm{~A}}$ expression in sec14-1 ${ }^{\text {ts }}$ sac1 mutants effected significant enhancements in Ptdlns-4-P levels at $37^{\circ} \mathrm{C}$ (Figure 4B). Ptdlns-4-P levels were indifferent to Sfh1 expression.

\section{Ptdlns and PtdCho binding are required for manifestation of SFH1 * phenotypes}

Individual Ptdlns- and PtdCho-binding activities are essential for productive Sec14 biological activity, and these cooperate in stimulating Ptdlns 4-OH kinase activity in vivo via a mechanism proposed to involve Sec14mediated Ptdlns-presentation to lipid kinase (Schaaf et al., 2008; Bankaitis et al., 2010). To determine whether PL binding in general is required for elaboration of Sec14-like activities by Sfh1*, "pinchclose" mutants that abrogate binding of all PLs were incorporated into the context of several SFH1* alleles. Residues $L_{179}, I_{196}$, and $V_{199}$ organize a region of the Sfh1 hydrophobic cavity that defines PtdCho and Ptdlns acyl chain-binding space. Thus introduction of bulkier amino acids (e.g., W) at these positions results in steric incompatibility with PL binding by Sfh1 or Sec14 (Schaaf et al., 2008). As expected, recombinant Sfh 1 Y113C,L179W,I196W was defective in 
A

7.5

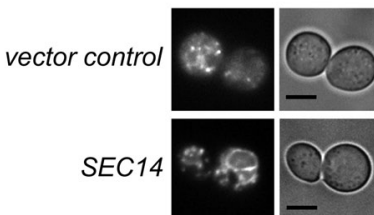

E126A

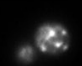

10

Y109C

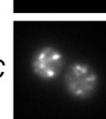

Y113C
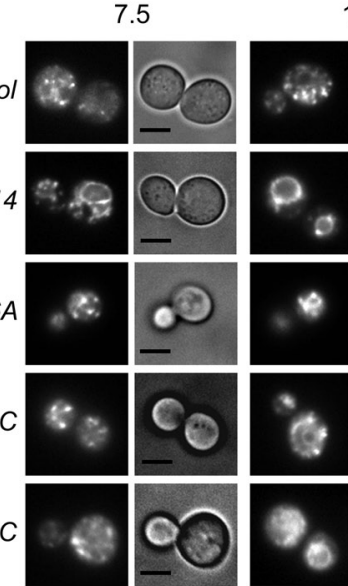

15
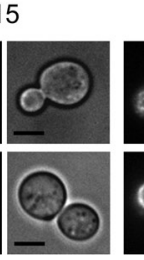

30

[min]
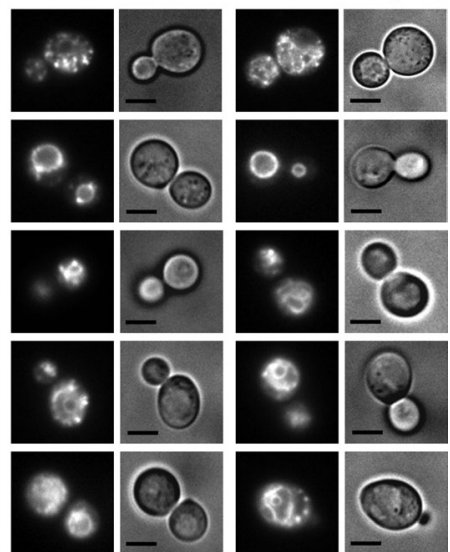

B

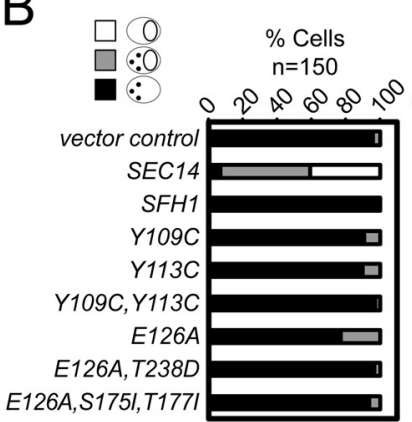

7.5
$\%$ Cells

$\mathrm{n}=150$
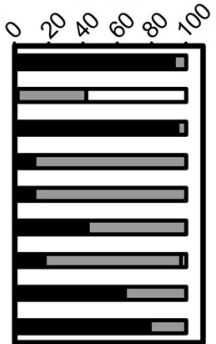

15
$\%$ Cells

$\mathrm{n}=150$
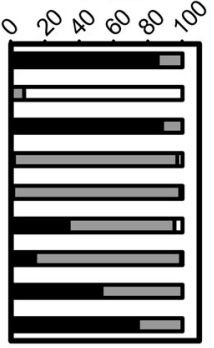

30 [min]
FIGURE 3: Sfh1* and endocytic membrane trafficking from the plasma membrane to the vacuole. A sec14-1 ${ }^{\text {ts }}$ yeast strain (CTY1-1A) carrying the indicated YCp expression plasmids (top) were cultured at $25^{\circ} \mathrm{C}$ to early logarithmic growth phase in uracil-free minimal medium and shifted to $37^{\circ} \mathrm{C}$ for $2 \mathrm{~h}$. Cells were then pulsed with FM4-64 (10 $\mu \mathrm{M}$ for $10 \mathrm{~min})$, rapidly sedimented, and reconstituted in fresh medium without FM4-64 to initiate chase. Aliquots were removed at the indicated times of chase, poisoned with a $\mathrm{NaN}_{3} / \mathrm{NaF}$ cocktail (final concentration of $10 \mathrm{mM}$ each), and kept on ice in the dark before visualization. FM4-64 profiles were imaged using a Nikon E600 fluorescence microscope. The YCp(URA3) and YCp(SEC14) conditions represent negative and positive controls, respectively. (A) Representative images are shown. Bar $=5 \mu \mathrm{m}$. (B) Distributions of imaging profiles (key shown at left) are quantified as \% of total cells analyzed ( 150 for each condition). Quantitative chase of FM4-64 from the plasma membrane into the terminal vacuolar compartment is depicted in white, partial delivery in gray, and strictly endosomal localization of tracer in black.

PL-transfer activity (Supplemental Figure S3, A and B), and the triple mutant proteins were stable in vitro and in vivo (Supplemental Figure S3, C and D). Neither expression of Sfh $1{ }^{\mathrm{Y} 113 C, L 179 W, 1196 \mathrm{~W}}$, nor of Sfh1Y113C,L179W,V199W, rescued growth defects associated with the sec $14-1^{\text {ts }}$ allele (Figure $4 \mathrm{C}$ ). The Sfh $1^{\mathrm{E} 126 \mathrm{~A}}$ pinch-close derivative was also generated, but this triple mutant protein was unstable in vivo (unpublished data).

Incorporation of $\mathrm{T}_{238} \mathrm{D}$ into the Sfh1 ${ }^{*}$ context, that is, a substitution that specifically interferes with coordination of the Ptdlns headgroup phosphate (Schaaf et al., 2008), evoked the expected ablation of Ptdlns-transfer activity (Supplemental Figure S3A). Sfh 1 ${ }^{\mathrm{Y} 113 \mathrm{C}, T 238 \mathrm{D}}$ and Sfh1 12126A,T238D were both stable polypeptides in vivo and in vitro (Supplemental Figure S3, C and D), and expression of these proteins failed to rescue growth defects of sec14-1 $1^{\text {ts }}$ yeast at restrictive temperatures (Figure $4 \mathrm{C}$ ). Sfh1 residues $S_{175}$ and $T_{177}$ play important roles in coordinating the PtdCho headgroup phosphate within the Sfh1 binding pocket, and combinatorial substitution of these residues with bulky aliphatic amino acids (e.g., I) is nonpermissive for PtdCho binding (Schaaf et al., 2008). The PtdCho-

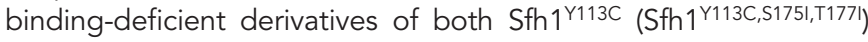
and Sfh1E126A (Sfh1 E126A,S175I,T177l) were stable polypeptides (Supplemental Figure S3, C and D), and in vitro PL-transfer assays confirmed the expected biochemical defects for these two proteins. Sfh1 ${ }^{\text {Y113C,S175I,T177l }}$ exhibited a > 25-fold reduction in PtdCho-transfer activity relative to $S f h 1^{Y 113 C}$ and retained Ptdlns transfer activity (Supplemental Figure S3, A and B). Expression of Sfh 1 Y113C,S175I,T177। or Sfh1 1 126A,S1751,T1771 from low-copy vectors failed to rescue growth of sec14-1 $1^{\text {ts }}$ strains at restrictive temperatures (Figure 4C). Moreover, enhanced expression of Sfh1 ${ }^{\text {Y113C,S175I,T1771 }}$ or Sfh1E126A,S1751,T1771 driven by high-copy vectors failed to rescue lethality associated with the sec14 $\Delta$ allele (Figure 4C).

The failures of the various PL-binding-defective variants of SFH1* to rescue sec14-1 $1^{\text {ts }}$-associated growth defects translated to defects in regulation of PIP homeostasis. Stable variants of Sfh1* impaired for PL binding (Sfh1Y113C,L179W,1196W), specifically defective in either

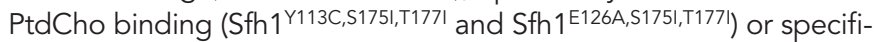
cally defective in Ptdlns binding (Sfh $1^{\text {Y113C,T238D }}$ and Sfh 1 ${ }^{\text {E126A,T238D), }}$ were all incompetent for augmentation of Ptdlns-3-P, Ptdlns-4-P, or

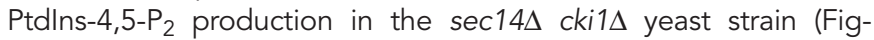
ure 4A). As expected, in every case, Ptdlns and PtdCho binding were each individually required for $\mathrm{Sfh} 1{ }^{*}$-dependent rescue of CPY transit through the TGN/endosomal system (unpublished data).

\section{SFH1* alleles and polar interactions in the hydrophilic microenvironment}

The data suggest a relationship between Sfh 1 * mechanisms and the functional status of the enigmatic hydrophilic patch. The $Y_{109}$ $\rightarrow \mathrm{F}, \mathrm{C}, \mathrm{H}, \mathrm{A}$; the $\mathrm{Q}_{111} \rightarrow \mathrm{A}$; the $\mathrm{Y}_{113} \rightarrow \mathrm{C}$; and the $\mathrm{E}_{126} \rightarrow \mathrm{A} \mathrm{Sfh}^{*}$ * substitutions are all predicted to weaken polar contacts within this polar motif. In this regard, the $\mathrm{Y}_{109} \mathrm{~F}$ substitution maintains the shape and size of the side chain but abolishes the ability to establish side chain hydrogen bonds by lack of the phenolic hydroxyl group. We therefore tested whether Sfh ${ }^{*}$ substitutions exhibited allele specificities consistent with weakened polar interactions within this motif. Indeed, in contrast to the cognate Sfh1 ${ }^{\text {E126A }}$ example, Sfh1E126D (i.e., protein in which polar contacts within the hydrophilic patch are presumably conserved) presented severely attenuated Sfh 1 * phenotypes-despite its in vivo stability (Supplemental Figure S4, A and B). The residual Sfh 1* character associated with the $E_{126} D$ substitution suggests that the length of the side chain (and thus spacing within the hydrophilic patch) contributes to these polar interactions.

Sfh 1 and Sec14 share substantial primary sequence conservation within the hydrophilic patch. Indeed, most residues within a 4-Å radius of Sfh1 residue $Y_{109}$ are conserved between the two proteins. These conservations include Sfh1 (Sec14) residues: $A_{106}\left(A_{104}\right), K_{107}$ $\left(K_{105}\right), Y_{109}\left(Y_{107}\right), P_{110}\left(P_{108}\right), Q_{111}\left(Q_{109}\right), E_{126}\left(E_{124}\right), E_{127}\left(E_{125}\right), L_{128}$ $\left(L_{126}\right), M_{145}\left(M_{143}\right)$, and $Y_{195}\left(Y_{193}\right)$. Sfh $1^{Y 195 A}$ was of interest because the corresponding substitution interferes with cooperative interactions between residues $Y_{195}$ and $Y_{109}$-that is, interactions that organize polar contacts with ordered water in the hydrophilic patch. When tested in both high- and low-copy expression contexts, Sfh $1^{\text {Y195A }}$ exhibited Sfh1* properties (unpublished data). These results are congruent with the idea that strong polar interactions within the hydrophilic patch are incompatible with Sfh1*

Primary sequence divergences between Sfh1 and Sec14 in the hydrophilic region include $L_{105}\left(I_{103}\right), M_{108}\left(F_{106}\right)$, and $I_{131}\left(V_{129}\right) . M_{108}$ 


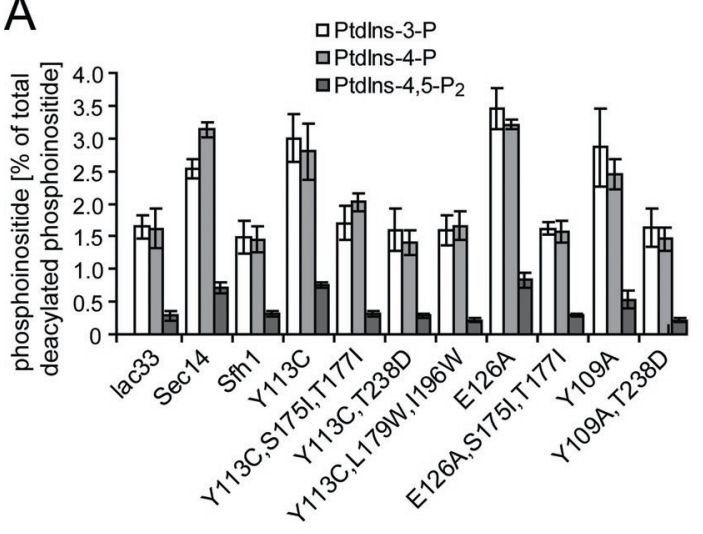

C

vector control
SEC14
SFH1
Y113C
Y113C,S175I, T177I
Y113C, T238D
Y113C,L179W, I196W
Y113C,L179W,V199W
E126A
E126A,S175I, T77I
E126A, T238D
Y109A
Y109A,T238D
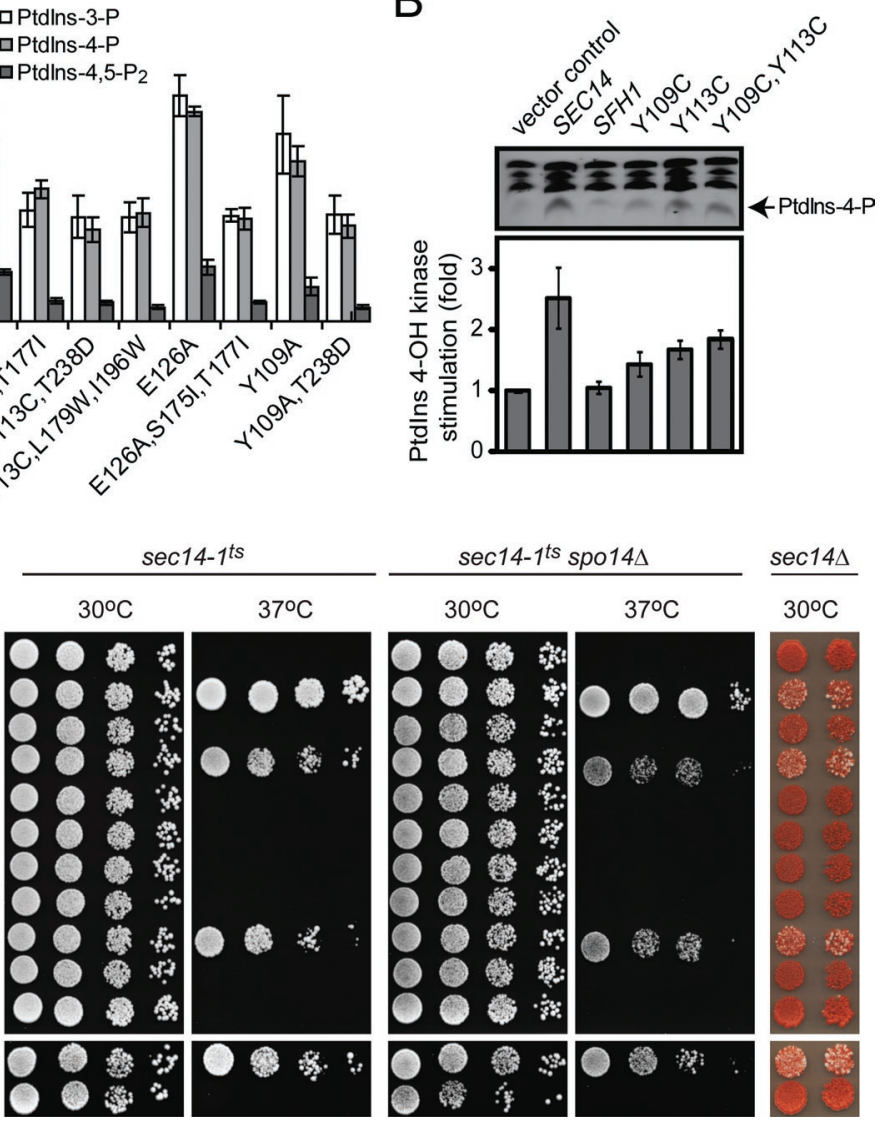

FIGURE 4: Potentiation of Ptdlns 4-OH kinase activities. (A) The sec14 $\Delta$ cki1 "bypass Sec14" yeast strain CTY303 was transformed with YCp(URA3) plasmids carrying SEC14, SFH1, or the designated $\mathrm{SFH}^{*}$ alleles. YCp(URA3) derivatives served as a negative control. Transformants were radiolabeled at $30^{\circ} \mathrm{C}$ for $12 \mathrm{~h}$ with $20 \mu \mathrm{Ci} / \mathrm{ml}\left[{ }^{3} \mathrm{H}\right]$ myo-inositol. Deacylated PIPs were separated by high-performance liquid chromatography and quantified (see Materials and Methods). Ptdlns-3-P, Ptdlns-4-P, and Ptdlns $(4,5) \mathrm{P}_{2}$ are indicated. Average values and SD are presented $(n=4)$. (B) Strain CTY100 (sec14-1's sac14; Cleves et al., 1989) carrying YCp plasmids for expression of the indicated genes were radiolabeled to steady state with $\left[{ }^{3} \mathrm{H}\right]$ inositol (Schaaf et al., 2008; lle et al., 2010). After a 3-h shift to $37^{\circ} \mathrm{C}$, phospholipids were extracted and resolved by TLC (top). Ptdlns-4-phosphate species is identified at left. The Ptdlns-4-phosphate band intensities were measured by densitometry and expressed as a Ptdlns-4-phosphate/Ptdlns ratio for purposes of normalization. The normalized ratio was then compared with the URA3 control condition (set to 1.0 on the relative scale). The data represent the averages and SD obtained from at least three independent experiments. (C) Left and middle, derivatives of isogenic sec14-1 $1^{\text {ts }}$ and sec14-1 $1^{\text {ts }}$ spo $14 \Delta$ yeast strains (as indicated on top) carrying designated YCp(URA3) plasmids were spotted in eightfold serial dilutions onto YPD agar plates.

Complementation of growth defects at $37^{\circ} \mathrm{C}$ reports Sec14-like in vivo function. Right, an ade2 ade3 sec14 4 yeast strain carrying a YEp(SEC14, LEU2, ADE3) plasmid (strain CTY558; see Materials and Methods) was transformed with high-copy YEp(URA3) plasmids carrying SFH1 or the designated sfh1* alleles and dilution spotted onto YPD agar plates. Appearance of white segregant colonies that acquire leucine and histidine auxotrophies reports loss of parental YEp(SEC14, LEU2, ADE3) and hence indicates functionality of the mutant SFH1* gene product.

was excluded from analysis because structural models indicate the side chain protrudes away from the hydrophilic surface and is not involved in the polar interactions of interest. By contrast, $I_{131}$ (a residue identified as an Sfh1* hotspot; discussed previously) lies close to the $\mathrm{C}_{11}$ and $\mathrm{C}_{18}$ positions of the PtdCho and Ptdlns sn-2 acyl chains, respectively. $\mathrm{I}_{131}$ extends the hydrophilic patch boundary toward the headgroup-distal ends of bound PtdCho or Ptdlns sn-1 and sn-2 acyl chains (Supplemental Figure S4C). Sfh $1^{1131 V}$ expression rescued growth defects associated with sec14-1 ${ }^{\text {ts }}$ and the isogenic sec14-1 $1^{\text {ts }}$ spo14 $\Delta$ double mutant (Supplemental Figure S4A). $\underline{\sec 14 \Delta}$

Sfh1 ${ }^{\mathrm{E} 126 \mathrm{~A}}$ crystal structure

The crystal structure of Sfh $1{ }^{\mathrm{E} 126 \mathrm{~A}}$ in complex with a bound 16:0/18:0 phosphatidylethanolamine (PtdEtn) was determined to $1.8 \AA$ resolution (Supplemental Table S1). A struc-

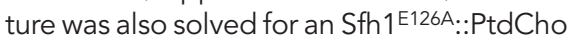
complex, but the resolution was inferior $(2.8 \AA)$. For this reason, and because both amino-PLs occupy the same site within the Sfh1 hydrophobic pocket (Schaaf et al., 2008), the PtdEtn-bound complex was analyzed. Sfh 1 E126A::PtdEtn displays the canonical closed conformation that superimposes onto the Sfh1::PtdEn structure with a root mean square deviation (rmsd) of $0.15 \AA$ for all protein atoms. In the Sfh1::PtdEn complex, the $E_{126}$ side chain makes direct contacts with $Y_{109}$ and $Y_{124}$, and $Y_{124}$ participates in an $\mathrm{H}$-bond interaction with $\mathrm{Q}_{111}$ (Figure 5B). Atomic displacement parameters for these residues are 16.8, 17.2, and $16.9 \AA^{2}$ in the Sfh1::PtdEn complex and $17.7,17.9$, and $16.9 \AA^{2}$ in the Sfh1 1 E126A.:PtdEn complex, respectively. As in the Sfh $1::$ PtdEn complex (Schaaf et al., 2008), PtdEtn is stabilized by 14 van der Waals interactions and coordinated via hydrogen bonds by residues $\mathrm{Y}_{113}$ and $\mathrm{S}_{175}$ and by one $\mathrm{H}_{2} \mathrm{O}$ molecule (Figure 5C).

Inspection of the ligand-binding pocket reveals that the primary difference between Sfh 1 and Sfh $1{ }^{\mathrm{E} 126 \mathrm{~A}}$ is the configuration of ordered $\mathrm{H}_{2} \mathrm{O}$ that fills the hydrophilic patch directly underneath the bound PL. In Sfh1, the $E_{126}$ side chain carboxylate moiety engages in strong $\mathrm{H}$-bond interactions with residues $Y_{109}$ and $Y_{124}$ and is part of a larger $\mathrm{H}$-bond network that extends to residue $\mathrm{Y}_{195}$ and involves another conserved $\mathrm{H}_{2} \mathrm{O}$. The $\mathrm{E}_{126}$ side chain carboxylate interaction is replaced by an $\mathrm{H}_{2} \mathrm{O}$ in the $\mathrm{Sfh} 1{ }^{E 126 A}$ :: PtdEn complex (Figure 5, A and B), a configuration that preserves the $\mathrm{H}$-bonding network and hydrophilic character of this region of the hydrophobic pocket. The $\mathrm{H}_{2} \mathrm{O}$ molecule coordinated by residues $Y_{109}$ and $Y_{195}$ displays atomic displacement parameters of 9.8 and $23.2 \AA^{2}$ in the Sfh1 1 E126A.:PtdEn and Sfh $1:$ :PtdEtn complexes, respectively. These data report that $\mathrm{E}_{126} \mathrm{~A}$ causes a remarkably subtle destabilization of the hydrophilic patch and suggest altered water behavior in this region is a primary contributor to $\mathrm{Sfh} 1{ }^{*}$ phenotypes (discussed later in this article).

\section{Sfh1* and PtdCho binding/exchange}

While the structural data suggest $\mathrm{E}_{126} \mathrm{~A}$ relieves the $\mathrm{Y}_{109}$ side chain from its normally strong interaction with $E_{126}$ in favor of an interaction with this newly positioned $\mathrm{H}_{2} \mathrm{O}$ molecule, the nearly complete structural identity between Sfh1 and Sfh 1 E126A otherwise offers little insight into why the $E_{126} A$ resuscitates Sec14-like activities in Sfh1. One plausible mechanism is that Sfh $1^{*}$ are endowed with superior 

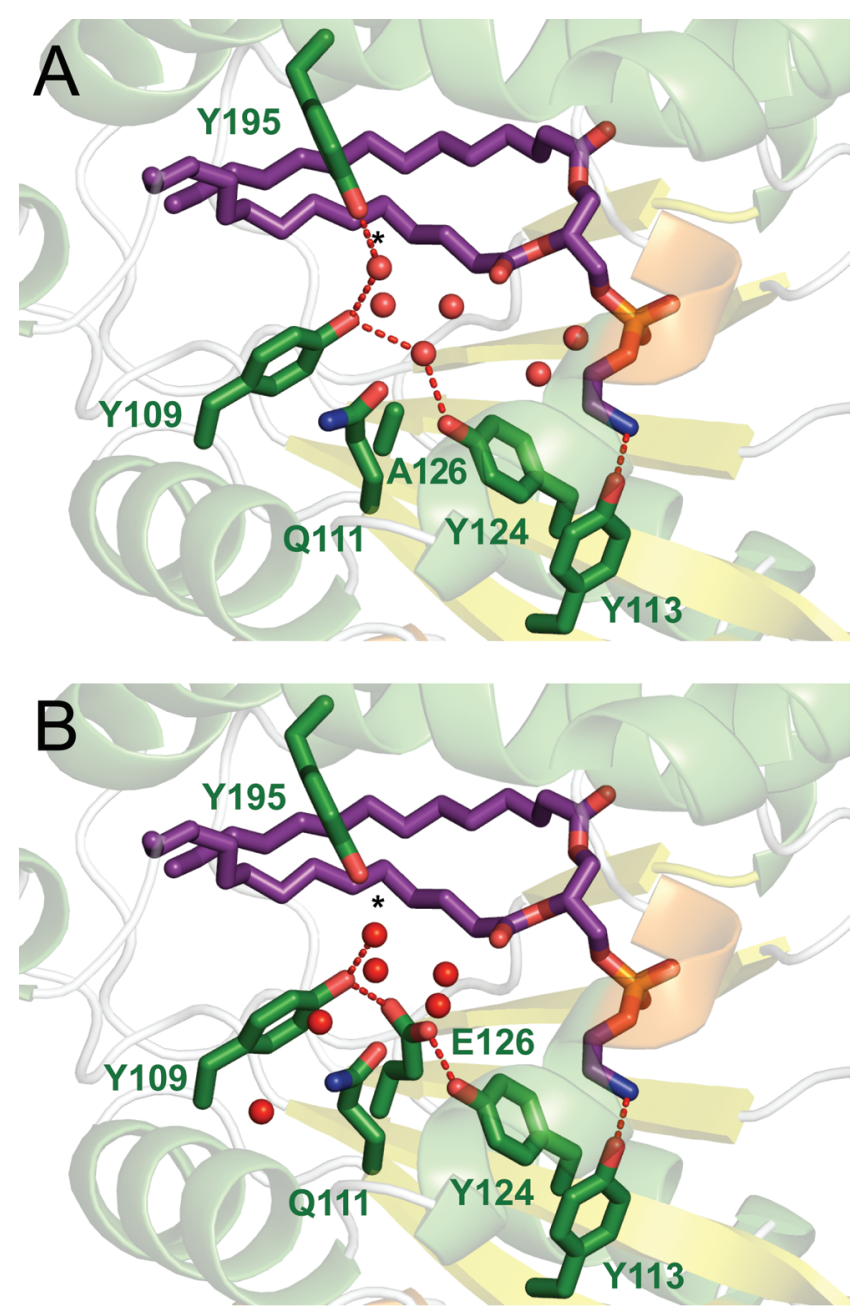

C $\mathrm{V} 230 \mathrm{O}$ F223 $\mathrm{L}$ L234 O

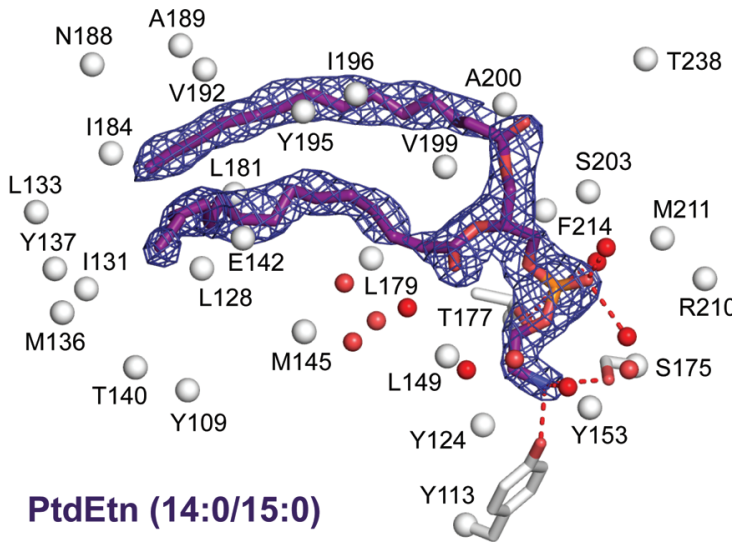

FIGURE 5: Crystal structure of Sfh1 12126A bound to PtdEtn. The hydrophilic patch in (A) Sfh1E126A::PtdEtn and (B) Sfh1::PtdEtn (pdb 3B74). The patch is formed by residues $Y_{109}, Q_{111}, Y_{113}, Y_{124}$, and $E_{126}$. The protein atoms are rendered as green sticks, bound PtdEtn is depicted as purple sticks, ordered waters are depicted as red spheres, and $\mathrm{H}$-bonds are shown as dashed lines. The sn-2 acyl chain C5 position is highlighted by *. (C) $2 \mathrm{~F}_{\mathrm{O}}-\mathrm{F}_{\mathrm{C}}$ composite omit electron density (contoured at $1 \sigma$ ) for the bound PtdEtn. Residues within $4.2 \AA$ of the bound PL are shown as white spheres, and waters are shown as red spheres. Residues that coordinate the headgroup moieties are shown as sticks, with $\mathrm{H}$-bonds represented as dashed lines. capacities to cycle PL into and out of the hydrophobic pocket. To monitor protein::PL interactions directly, continuous wave (CW) electron paramagnetic resonance (EPR) spectroscopy was used to compare the parameters of PtdCho binding by Sfh 1 and Sfh1*. Spin-labeled n-doxyl-PtdCho molecules were used to survey protein::PtdCho interactions across the lipid molecule. In these experiments, the EPR signal from liposomal n-doxyl-PtdCho species appears as a broad single line of 25-27 G peak-to-peak width. Such a spectrum is readily modeled by a Lorentzian function and reflects the strong dipole-dipole and exchange interactions that occur when nitroxide moieties are packed in close proximity. On sequestration of n-doxyl-PtdCho within the Sfh1 or Sfh1* lipid-binding cavity, spin-spin interactions between $n$-doxyl-PtdCho molecules are eliminated with the result that a sharper EPR spectrum is produced.

Figure 6A displays the CW X-band spectra recorded when individual n-doxyl-PtdCho species were incubated with Sfh1, Sfh1 ${ }^{\mathrm{Y} 113 \mathrm{C}}$, or Sfh 1 ${ }^{\mathrm{E} 126 \mathrm{~A}}$. For all spin probe positions analyzed, the contribution of liposomal n-doxyl-PtdCho registered as a broad line most readily observed at the wings. Contributions from the liposomal n-doxylPtdCho were subtracted from the experimental spectra, yielding the component corresponding to protein-bound n-doxyl-PtdCho. Relative contributions from protein-bound and liposomal forms were quantified by a double integration of the corresponding spectral components. These analyses demonstrate Sfh1 fails to incorporate 5- and 7-doxyl-PtdCho, while only poor binding was measured for the 10- and 12-doxyl-PtdCho; only $\sim 1-2 \%$ of the Sth1 molecules incorporated those spin-labeled species (Figure 6A, left; Figure 6B). 16-Doxyl-PtdCho was also inefficiently incorporated by Sfh1. Some $20 \%$ of total Sfh1 loaded with this spin-labeled PtdCho. These Xband CW EPR data demonstrate Sfh1 exhibits poor loading capacities for all n-doxyl-PtdCho-binding substrates.

By contrast, both Sfh 1 * proteins showed enhanced capacities for n-doxyl-PtdCho incorporation. For Sfh $1^{\mathrm{Y} 113 \mathrm{C}}$, weak incorporation was observed for 5- and 7-doxyl-PtdCho (10-15\% of total protein loaded), moderate incorporation was measured for 10- and 12-doxylPtdCho (30-40\% of total protein loaded), and Sfh $1{ }^{\mathrm{Y} 113 \mathrm{C}}$ was quantitatively loaded with 16-doxyl-PtdCho (Figure 6A, right; Figure 6B). Sfh $1{ }^{\mathrm{E} 126 \mathrm{~A}}$ was more impressive with regard to its capacity for $n$-doxylPtdCho incorporation. This protein was quantitatively occupied with 5-doxyl-PtdCho and demonstrated significant incorporation of 7-, $10-, 12-$, and 16-doxyl-PtdCho as well (40-50\% of total protein loaded across the range of probe positions; Figure 6A, center; Figure 6B). By comparison, Sec14 is quantitatively loaded with each of the $n$-doxyl-PtdCho species under these experimental conditions (Figure 6B; Smirnova et al., 2006; Smirnova et al., 2007). The acquired capability of Sfh 1 * to load with $n$-doxyl-PtdCho into the hydrophobic pocket is not likely due to relief of steric problems associated with accommodating the spin label within the hydrophobic pocket. The effects were scored even when the spin-label position was physically distant from the operant $\mathrm{Sfh} 1{ }^{*}$ substitution.

The EPR data project that Sfh $1{ }^{*}$ substitutions are associated with enhanced cycling of $\mathrm{PL}$ into and out of the protein interior. We therefore expected that Sfh ${ }^{*}$ proteins would exhibit increased specific activities of Ptdlns and/or PtdCho transfer relative to Sfh1. This prediction was tested by comparing the Ptdlnsand PtdCho-transfer activities of Sfh $1{ }^{*}$ to those of purified Sec14 and Sfh1. All proteins were stable during the course of the PLtransfer experiments (Supplemental Figure S3C), thereby permitting quantitative comparisons. His 8 -Sec14 exhibited robust Ptdlns- and PtdCho-transfer activities, whereas His${ }_{8}-\mathrm{Sfh} 1$ showed $>$ fivefold reductions in specific activities for Ptdlns and PtdCho 
A

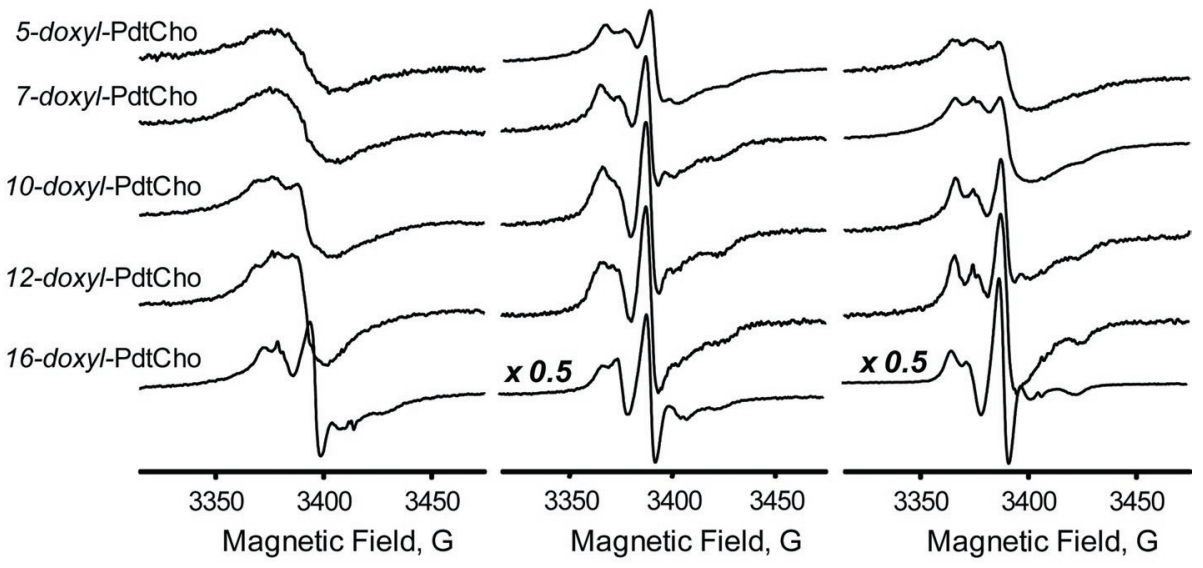

B

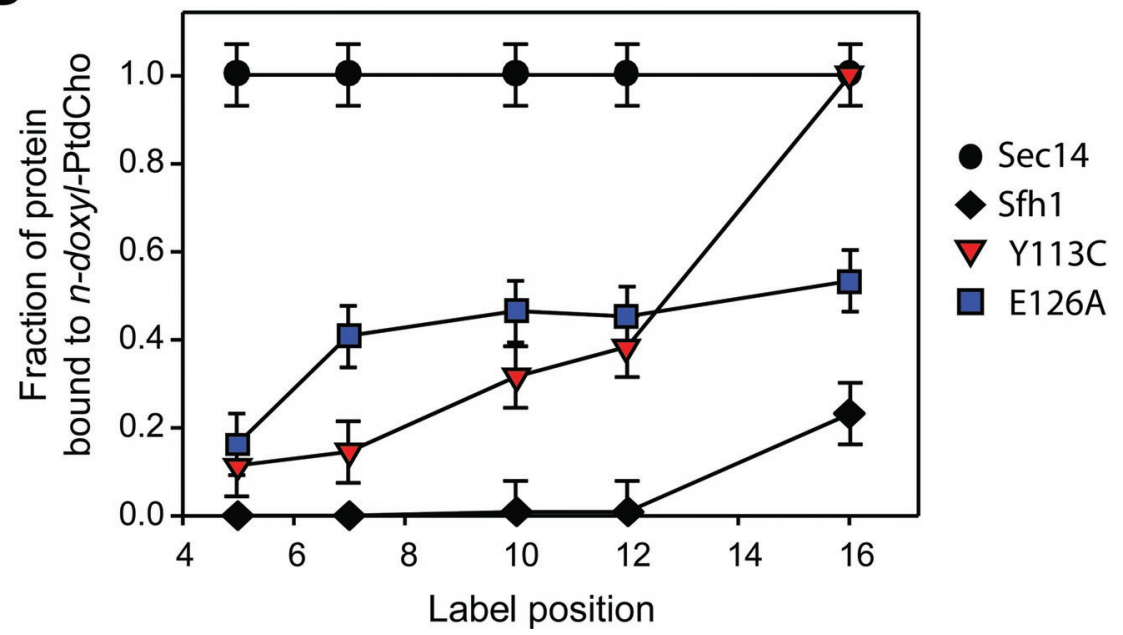

FIGURE 6: Spin-labeled PtdCho-binding parameters for Sfh1* proteins. (A) Room temperature $\left(\mathrm{T}=23^{\circ} \mathrm{C}\right) \mathrm{X}$-band CW EPR spectra from $n$-doxyl-PtdCho prepared as multilamellar lipid dispersion after mixing with Sfh1 protein and its mutants in 2:1 lipid-to-protein molar ratio (left, Sfh1; center, Sfh1 ${ }^{\mathrm{E} 126 \mathrm{~A}}$; right, Sfh1 ${ }^{\mathrm{Y} 113 \mathrm{C}}$ ). Modulation amplitude is $0.5 \mathrm{G}$, and spectra are intensity normalized using double integration. (B) Binding of $n$-doxyl-PtdCho to protein as a function of the label position along the sn-2 acyl chain after mixing n-doxyl-PtdCho prepared as multilamellar lipid dispersion with proteins in $\sim 2: 1$ lipid-to-protein molar ratio. Fraction of protein containing n-doxyl-PtdCho bound: circles, n-doxyl-PtdCho bound to Sec14; diamonds, n-doxylPtdCho bound to Sfh1; triangles, n-doxyl-PtdCho bound to Sfh1 ${ }^{\text {Y113C; }}$ squares, n-doxyl-PtdCho bound to Sfh1 1 126A.

transfer relative to $\mathrm{His}_{8}$-Sec14 (Supplemental Figure S3, A and B). All three $\mathrm{Sfh}_{1}{ }^{*}$ tested $\left(\mathrm{His}_{8}-\mathrm{Sfh} 1^{\mathrm{Y} 109 \mathrm{~A}}, \mathrm{His}_{8}-\mathrm{Sfh}{ }^{\mathrm{Y} 113 \mathrm{C}}{ }^{\text {, and } \mathrm{His}_{8}-}\right.$ Sfh1 ${ }^{\mathrm{E} 126 \mathrm{~A}}$ ) presented increased Ptdlns transfer activity relative to $\mathrm{His}_{8}$-Sfh1. Similar outcomes were recorded for PtdCho-transfer activities of $\mathrm{His}_{8}-\mathrm{Sfh} 1^{\mathrm{Y} 109 \mathrm{~A}}$ and $\mathrm{His}_{8}-\mathrm{Sfh} 1^{\mathrm{Y} 113 \mathrm{C}}$. Both $\mathrm{Sfh} 1^{*}$ proteins exhibited a $>$ fivefold increase in PtdCho transfer relative to $\mathrm{His}_{8}$-Sfh1. His 8 -Sfh1 ${ }^{\mathrm{E} 126 \mathrm{~A}}$ presented a twofold increase in PtdChotransfer activity relative to $\mathrm{His}_{8}-\mathrm{Sfh} 1$ (Supplemental Figure S3, A and B). Taken together, these data demonstrate that Sfh 1 * exhibit significantly enhanced (i.e., Sec14-like) abilities to cycle PL from the lipid-binding pocket relative to Sfh1.

\section{Molecular dynamics (MD) simulations}

Because the transition pathway between "closed" and "open" forms of the Sec14/Sfh1 fold that accompanies PL exchange cannot be directly monitored experimentally, MD simulations were used to model how Sfh1* conformational dynamics may differ from those of Sfh1 and more closely resemble those of Sec14. Unrestrained MD simulations were carried out with PL-bound Sfh1 and models of PL-

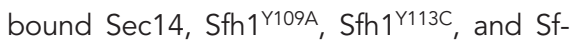
h1E126A. For the Sfh1E126A ::PL structures, Ptdlns and PtdCho were modeled into the crystal structure of Sfh1 ${ }^{E 126 A}:$ :PtdEtn (see Supplemental Materials). Surveys of the rmsd values of backbone atoms in snapshots written every 2 ps, during the course of three independent simulations, indicated that all systems consistently reached equilibrium within the 14-ns production run (Supplemental Figure S5). All independent simulations exhibited fluctuations $(\Delta \mathrm{rmsd})<$ $0.8 \AA$ within the last $2 \mathrm{~ns}(12-14 \mathrm{~ns})$. Unless otherwise noted, this interval was analyzed.

The primary difference between Sfh $1:: \mathrm{PL}$ structures and a previously crystallized apoSec14 is the repositioning of the Sfh $1 \mathrm{~A}_{9} / \mathrm{T}_{3}$ structural element, which controls access of $\mathrm{PL}$ to the hydrophobic pocket $\left(\mathrm{A}_{10} / \mathrm{T}_{4}\right.$ in Sec14; Sha et al., 1998; Ryan et al., 2007; Schaaf et al., 2008). The large motions of this helical gate, which occur in the transitions between open and closed Sec14 conformers, are essential for PL exchange and in vivo activity (Ryan et al., 2007). We were therefore interested in simulating the conformational transitions that occur when PLbound Sec14, Sfh1, or Sfh1* are used as starting structures. To this end, root mean square fluctuation (rmsf) of $\mathrm{C} \alpha$ atoms of all protein residues were extracted from the MD simulations and analyzed. In the case where dynamics of the open PL-free Sec14 starting structure were simulated, rmsf values exceeded $5 \AA$ for helical gate residues ${ }_{229} \mathrm{KPFLD}_{233}$ (Ryan et al., 2007). The corresponding rmsf values for all PL-bound starting structures remained $<2.5 \AA$, however (Figure 7). Thus the large conformational transitions required for PL exchange do not occur in PL-bound Sec14, Sfh1, or Sfh1* within the time frame of our MD simulations. This is not surprising given that the simulations were performed in a virtual aqueous environment with no membrane system to promote completion of the closed to open transition and to encourage egress of PL from the hydrophobic pocket.

Overall, Sec14-PL bound structures exhibited fluctuation profiles similar to those of their Sfh1 counterparts with rigid body motions of Sfh1 helices $A_{6}, A_{8}$, and $A_{9}$ (corresponding Sec14 helices are $A_{7}, A_{9}$, and $A_{10}$ ) (Figure 7). Fluctuations of the $A_{10}$ element of the Sec14 helical gate were obviously larger in both the Ptdlns and the PtdCho structures relative to fluctuations in the corresponding $A_{9}$ element of the Sfh1 and Sfh1* helical gate (Figure 7). Likewise, the Sec14 Aq helix exhibited increased fluctuations in both PL-bound structures as compared with the corresponding helix $A_{8}$ in Sfh1 and Sfh1* —with

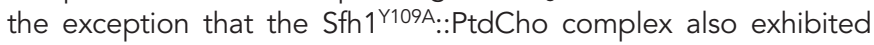
similarly enhanced fluctuations in the $A_{8}$ helix (Figure 7). Because 

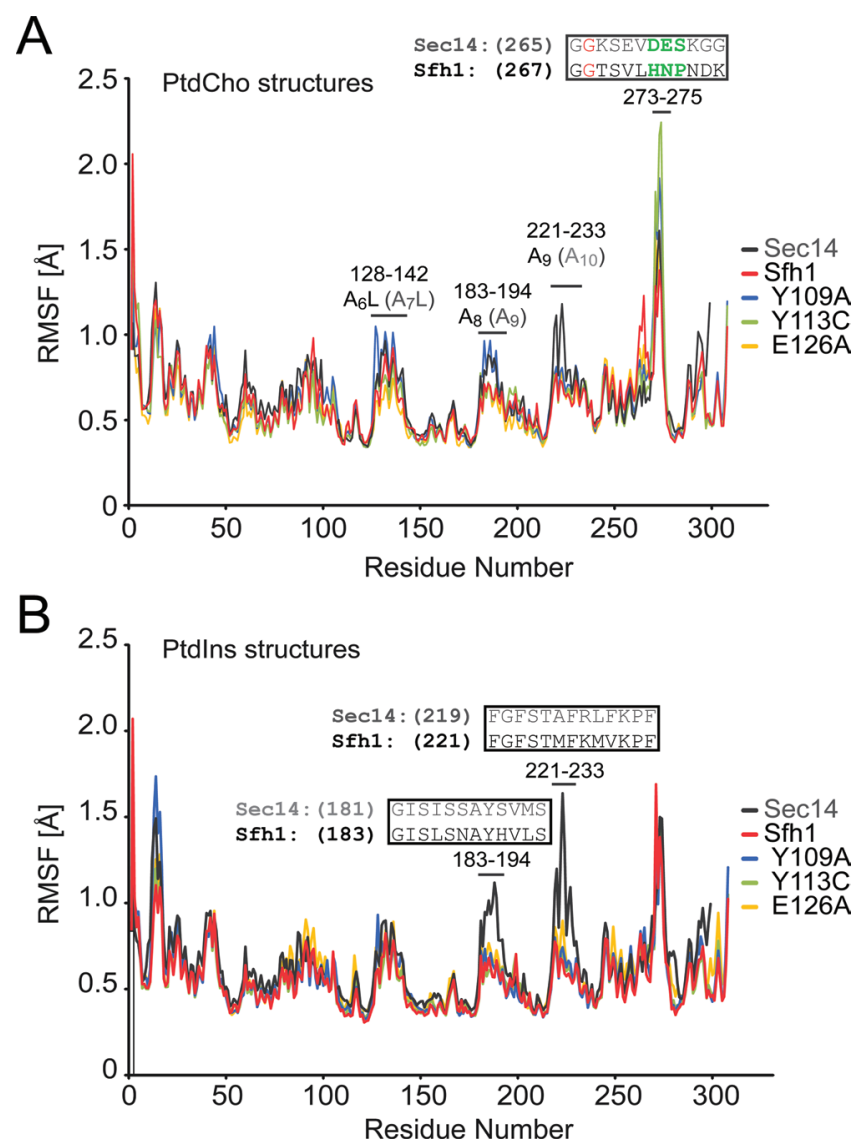

FIGURE 7: Conformational transitions of PL-bound structures. (A and B) Root mean square fluctuation (rmsf) values of $\mathrm{C} \alpha$ atoms of all protein residues were extracted from MD simulations of PtdCho (A) and Ptdlns structures (B). Average $\mathrm{C} \alpha$ atomic position fluctuations from three simulations are plotted as a function of residue number ( $\mathrm{N}$ to $\mathrm{C}$ terminus) for Sec14-PL, Sfh1-PL, and Sfh1*-PL (colors as indicated). Selected regions of high mobility are highlighted in black letters (Sfh1 and Sfh1*) or gray letters (Sec14). Sec14 residue $\mathrm{G}_{266}$ and corresponding Sfh 1 residue $\mathrm{G}_{268}$ are depicted in red, and the Sec14 ${ }_{271} \mathrm{DES}_{273}$ motif and corresponding Sfh1 ${ }_{273} \mathrm{HNP}_{275}$ motif are depicted in green (A).

Sfh1 helices $A_{8}$ and $A_{9}\left(\operatorname{Sec} 14 A_{9}\right.$ and $\left.A_{10}\right)$ are in intimate contact in the PL-bound conformation, we speculate that increased fluctuations in $\mathrm{Sfh} 1{ }^{\mathrm{Y} 109 \mathrm{~A}}$ helix $\mathrm{A}_{8}$ reflect enhancements in the initial motions of the conformational transition that results in opening of the helical gate, thereby accounting for the strong Sfh 1* phenotype associated with the $Y_{109} A$ substitution (Figure 7).

The MD simulations also consistently identified common Sfh1*specific and Sec14-like behaviors in key structural motifs. Sec14 residues ${ }_{271} \mathrm{DES}_{273}$ exhibit rmsf values of $>1.6 \AA$ in Sec14::PtdCho simulations. Interestingly, all Sfh $1 *::$ PtdCho simulations exhibited significantly enhanced fluctuations of the corresponding ${ }_{273} \mathrm{HNP}_{275}$ motif relative to the Sfh1::PtdCho control. The rmsf values for Sfh1 $1^{\mathrm{Y} 109 \mathrm{~A}}(1.9 \AA)$, Sfh $1^{\mathrm{Y113C}}(2.24 \AA)$, and Sfh $1^{\mathrm{E} 126 \mathrm{~A}}(1.6 \AA)$ are measured against rmsf values of $<1.4 \AA$ for this motif when Sfh $1::$ PtdCho was used as starting structure. These findings are of particular interest because 1) conformational transitions involving this specific motif closely correlate with dynamics of the helical gate in the open PLfree Sec14 structure (Ryan et al., 2007), and 2) this element lies in immediate proximity to the $\operatorname{Sec} 14 \mathrm{G}_{266} \mathrm{D}$ (i.e., sec14-1 ${ }^{\text {ts }}$ ) missense substitution $\left(\mathrm{G}_{268}\right.$ in $\left.\mathrm{Sfh} 1\right)$, which ablates both in vitro PL-exchange activity and in vivo Sec14 function at restrictive temperatures (Ryan et al., 2007).

\section{Altered interactions between the $B_{1} L_{2}$ and the $A_{11} L_{4}$ substructures in Sfh1*}

The Sec14 $B_{1} L B_{2}$ and $A_{12} L T_{5}$ substructures comprise a "gating module" that transduces conformational information to the $A_{10} T_{4} A_{11}$ helical gate via an extensive $\mathrm{H}$-bond network. The ${ }_{114} \mathrm{TDKDGR}_{119}$ component of the $\mathrm{B}_{1} \mathrm{LB}_{2}$ element plays a particularly important role in that conformational coupling (Ryan et al., 2007). The corresponding gating module elements in Sfh 1 are $\mathrm{B}_{1} \mathrm{LB}_{2}$ (including the ${ }_{116} \mathrm{VD}$ $\mathrm{KDGR}_{121}$ component) and $\mathrm{A}_{11} \mathrm{LT}_{4}$. Strikingly, the core $\mathrm{Sfh} 1^{*}$ residues lie immediately $C$-terminal $\left(Y_{109}\right)$ to or within $\left(Q_{111}, Y_{113}\right.$, and $\left.E_{126}\right)$ the $B_{1} L_{2}$ element, and these residues flank the ${ }_{116} V_{D K D G R} 121$ component. To investigate whether the $\mathrm{H}$-bonding network of residues comprising the hydrophilic patch is functionally involved in the Sfh $1^{*}$ phenotype, we searched for altered $\mathrm{H}$-bond interactions in this network in the context of Sfh $1^{*}$. Alterations of interest were defined by consistent absolute values of change of $>10 \%$ occupancy (i.e., the percentage of time an individual hydrogen bond is present) in all Sfh ${ }^{*}$ mutants relative to Sfh 1 . In the Sfh $1::$ PtdCho complex, 48 residues are in $\mathrm{H}$-bond contact with the core $\mathrm{Sfh} 1{ }^{*}$ residues (Supplemental Figure S6). Additional $\mathrm{H}$-bond interactions are observed with residues of the $A_{7} L A_{8}$ helical motif and residues of the $C$-terminal string motif (Supplemental Figure S6).

Increased $\mathrm{H}$-bonding occupancy was consistently recorded in all Sfh $1 *::$ PtdCho simulations for five $\mathrm{H}$-bond pairs. The most dramatic increase of $\mathrm{H}$-bonding was observed for the interaction of the $\mathrm{E}_{126}$ backbone with the phenolic $-\mathrm{OH}$ group of $\mathrm{Y}_{266}$. While the occupancy in Sfh $1::$ PtdCho simulations was $<9.5 \%$, it increased to $48 \%$ and $49 \%$ in the Sfh $1^{\mathrm{Y} 109 \mathrm{C}}$ and Sfh $1^{\mathrm{Y} 113 \mathrm{C}}$ contexts, respectively. The backbone $\mathrm{H}$-bonding interaction between residue $A_{126}$ and $Y_{266}$ in Sfh1 1 126A likewise exhibited a significant increase to $21 \%$ occupancy (Supplemental Table S2). The neighboring $\mathrm{E}_{127}-\mathrm{K}_{265}$ side chain interaction also increased robustly in all Sfh $1{ }^{*}$ simulations. While the side chain interaction showed an occupancy of $75 \%$ in the Sfh $1::$ PtdCho context, this value increased to $95 \%, 88 \%$, and $97 \%$ in the corresponding Sfh $1^{\mathrm{Y} 109 \mathrm{C}}$, Sfh $1^{\mathrm{Y} 113 \mathrm{C}}$, and Sfh1 ${ }^{\mathrm{E} 126 \mathrm{~A}}$ simulations, respectively (Supplemental Table S2). These data signify increased interactions between the $C$-terminal region of $B_{2}$ and the $T_{4}$ in the Sfh 1 *::PtdCho structures (Figure 8A; Supplemental Figure S6). Two potential consequences of those increased interactions include the following: 1) The loop region between the $B_{1}$ and the $B_{2}$ strands undergoes conformational changes (as evidenced by increased $\mathrm{H}$ bonding between loop residues $D_{117}$ and $R_{121}$ of the ${ }_{116} V_{D N D G R}$ component of the gating module; see Figure 8B and Supplemental Figure S6), and 2) the unstructured regions that flank the $T_{4}$ are brought into closer proximity to each other as indicated by increased side chain interactions between $\mathrm{E}_{260}$ and $\mathrm{S}_{270}$ (Figure 8, B and C; Supplemental Figure S6; Supplemental Table S2).

To address the functional relevance of the increased $E_{126}-Y_{266}$ interaction, the $Y_{266} F$ and $Y_{266} D$ substitutions were introduced into the context of Sfh $1{ }^{\mathrm{Y} 113 \mathrm{C}}$. These substitutions were anticipated to ablate $\mathrm{H}$-bonding by lack of the side chain $\mathrm{H}$-donor $\left(\mathrm{Y}_{266} \mathrm{~F}\right)$ for interaction with the $E_{126}$ backbone, or to cause an electrostatic clash $\left(Y_{266} D\right)$ with the terminal carboxylate of the $E_{127}$ side chain. Neither double mutant protein rescued sec14-1 $1^{\text {ts }}$-associated growth defects, even though the in vivo stability of each mutant polypeptide was preserved (Figure 8E). Thus increased interaction between the $\mathrm{N}$-terminal part of $\beta$-strand $B_{2}$ with the $T_{4}$ turn of substructure $A_{11} L T_{4}$ is a consistent, and functionally important, feature of the mechanisms underlying Sfh $1 *$. 
A
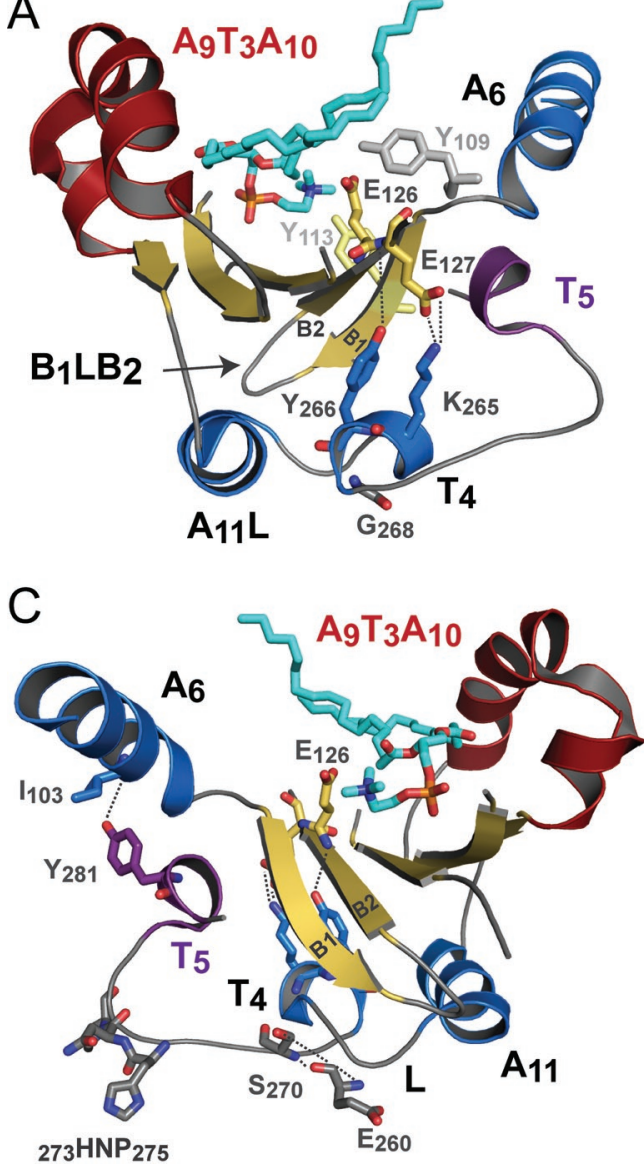

${ }_{273} \mathrm{HNP}_{275}$
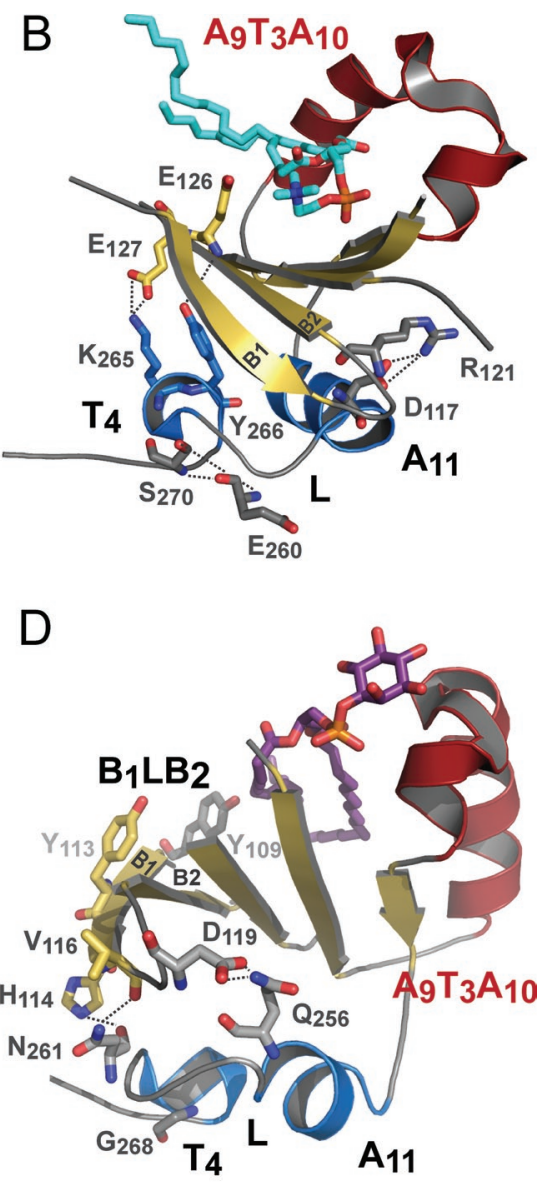

$E$

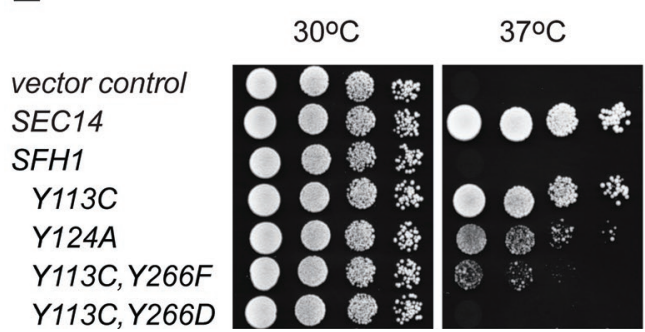

FIGURE 8: Model of Sfh1*-dependent alterations in H-bonding. (A-D) Ribbon diagrams illustrate $\mathrm{H}$-bonds that are altered consistently in Sfh1* mutants relative to Sfh1 (see Materials and Methods). The $A_{9} T_{3} A_{10}$ gating module is depicted in red, $T_{5}$ is depicted in magenta, and all other $\alpha$-helices are presented in blue. Unstructured regions are in gray, and $\beta$-strands are presented in yellow. PtdCho is shown as cyan stick (A and B) while Ptdlns is rendered in magenta (D). Relevant $\mathrm{H}$-bonds are presented as dashed lines. The $B_{1} L_{2}$ substructure consists of $\beta 1$ - and $\beta 2 \beta$-strands and the intervening loop region. The second substructure $A_{11} L T_{4}$ includes helix $A_{11}$ and extends to helix $\mathrm{T}_{4}$. Sfh $1^{*}$ mutants show increased interaction between the $\mathrm{C}$-terminal part of the $\beta 2$-strand and the $T_{4}$ turn (mediated by $\mathrm{H}$-bonding pairs $\mathrm{E}_{126}-\mathrm{Y}_{266}$ and $\mathrm{E}_{127}-\mathrm{K}_{265}, \mathrm{~A}$ ). Increased $\mathrm{H}$-bonding between $\mathrm{D}_{117}$ and $\mathrm{R}_{121}$, and between $\mathrm{E}_{260}$ and $\mathrm{S}_{270}$, are possible consequences of this interaction (B). Decreased H-bonding between $I_{103}$ and $Y_{281}$ provide a rational for increased fluctuations of the immediately $\mathrm{N}$-terminally positioned ${ }_{273} \mathrm{HNP}_{275}$ motif in the MD simulations $(C)$. Decreased $\mathrm{H}$-bonding of $A_{11} L T_{4}$ loop residue $N_{261}$ with $H_{114}$ and $V_{116}$ reduces interactions between the loop regions of the $B_{1} L B_{2}$ and $A_{11} L T_{4}$ substructures in Sfh1* mutants (D). (E) Serial dilution spotting of the sec14-1 $1^{\text {ts }}$ yeast strain transformed with YCp(URA3) plasmids carrying either SFH1 or the designated sfh1* alleles on YPD agar plates. Rescue at $37^{\circ} \mathrm{C}$ reports Sec14-like activity. YCp(URA3) and YCp(SEC14, URA3) plasmids served as negative and positive controls, respectively.

Consistent alterations in the $\mathrm{H}$-bond network between the $\mathrm{B}_{1} \mathrm{LB}_{2}$ and $\mathrm{A}_{11} \mathrm{LT}_{4}$ structural elements were also recorded in Sfh $1{ }^{*}:$ :Ptdlns simulations. Those alterations were characterized by reduced $\mathrm{H}$ - bonding occupancy between the loop regions of the $B_{1} L B_{2}$ and $A_{11} L T_{4}$ (Figure 8D; Supplemental Figure S7). Both elements strongly interact with each other in Sfh1 via $4 \mathrm{H}$-bonds: 1) the imidazole $\mathrm{NH}$ of $\mathrm{H}_{114}$ interacting with the $\mathrm{N}_{261}$ carbonyl, 2) a $\mathrm{Q}_{256}-\mathrm{D}_{119}$ side chain interaction, 3) a $\mathrm{Q}_{256}-\mathrm{D}_{117}$ side chain interaction, and 4) interaction of the $\mathrm{N}_{261}$ side chain with the $\mathrm{V}_{116}$ carbonyl. Three of these four interactions $\left(\mathrm{H}_{114}-\mathrm{N}_{261} ; \mathrm{Q}_{256}-\mathrm{D}_{119} ; \mathrm{V}_{116}-\mathrm{N}_{261}\right)$ are significantly reduced in Sfh1* (Supplemental Tables S2 and S3). The latter two involve the ${ }_{116} \mathrm{VDKDGR}_{121}$ component of the gating module-again emphasizing enhanced $B_{1} L_{2}$ dynamics as a mechanistic feature of the Sfh ${ }^{*}$ phenotype.

A consistent Sfh 1*-dependent decrease in $\mathrm{H}$-bond occupancy was also recorded for the interaction of the side chain carboxylate of activation residue $E_{126}$ with the phenolic hydroxyl group of $\mathrm{Y}_{124}$ (Supplemental Table $\mathrm{S} 2)$. That the reduced $\mathrm{H}$-bonding between these two residues contributes to the enhanced Sec14-like activities of Sfh1* is demonstrated by rescue of sec14-1 ${ }^{\text {ts }}$-associated growth defects by Sfh $1^{\mathrm{Y} 124 \mathrm{~A}}$ expression (Figure $8 \mathrm{E}$ ), even though $\mathrm{Sfh} 1^{\mathrm{Y} 124 \mathrm{~A}}$ shows decreased stability in vivo (unpublished data).

\section{Sfh1* enhance conformational fluctuations of the helical gate}

To assess the effects of Sfh 1 *-dependent alterations in the $\mathrm{H}$-bond network (which connects the $B_{1} L B_{2}$ and the $A_{11} L T_{4}$ substructures) on the dynamics of the helical gate, the interatomic distances between the $\mathrm{C} \alpha$ atoms of $Y_{190}\left(A_{8}\right)$ and $T_{225}\left(A_{9}\right)$ were monitored in Sec14::Ptdlns, Sfh $1::$ Ptdlns, and Sfh 1 *::Ptdlns structures, as were interatomic distances between $\mathrm{C} \alpha$ atoms of $\mathrm{K}_{197}\left(\mathrm{~A}_{8}\right)$ and $F_{233}\left(A_{9}\right.$; Figure $\left.9 A\right)$. Variations in this latter interatomic distance are useful for distance-monitoring analyses that report opening and closing of the helical gate in the apo-Sec14 molecule (residues $\mathrm{K}_{195}$ and $F_{231}$ in that context; Ryan et al., 2007). In agreement with the rmsf plots (Figure 7), $\mathrm{Y}_{190} \mathrm{~T}_{225} \mathrm{C} \alpha$ distance fluctuations were larger than those recorded for $\mathrm{K}_{197}-\mathrm{F}_{233}$ (Figure 9, B and C), suggesting that initial helical gate movements in the Ptdlns-occupied polypeptides are restricted mostly to the $\mathrm{C}$-terminal regions of helices $A_{9}$ and $A_{8}$. Interestingly, while rmsf values of $A_{9}$ and $A_{8}$ residues were indistinguishable between

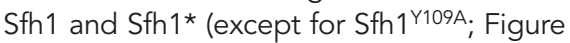
7), average fluctuations in the interatomic $\mathrm{Y}_{190} \mathrm{~T}_{225} \mathrm{C} \alpha$ distance were enhanced in all Sfh $1 *_{:: P t d l n s}$ structures relative to the Sfh $1::$ Ptdlns structure (Figure 9, B and C). These findings project that fluctuations in an Sfh ${ }^{*}$ context uniquely and productively translate into increased rigid body motions of helix $A_{9}$. These behaviors 
A

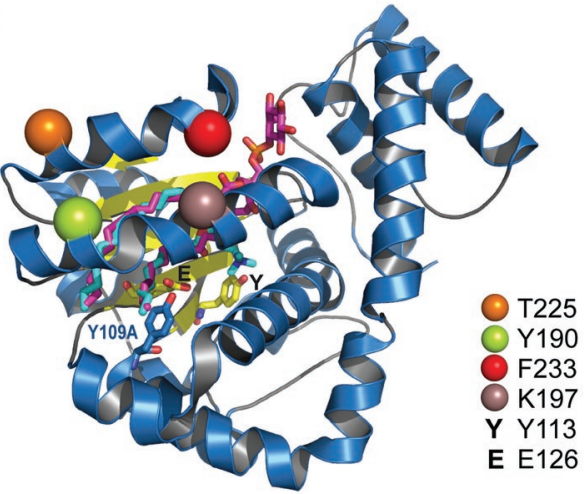

C

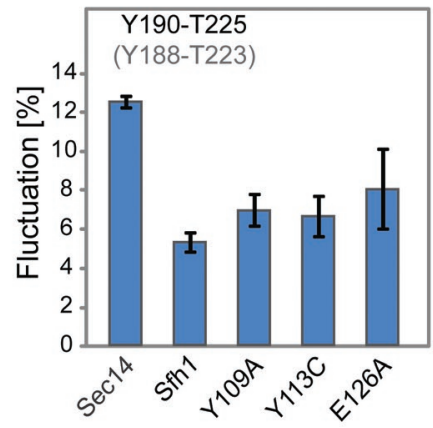

B

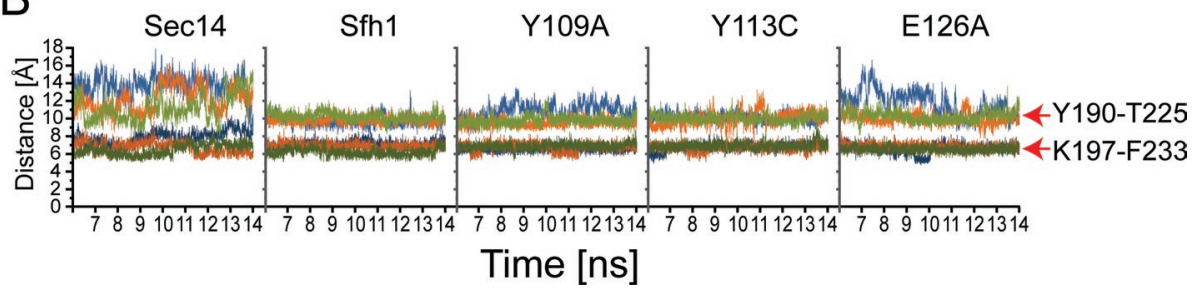

FIGURE 9: Helical gate motions and Sfh1*. (A) Ribbon diagram highlighting the positions of residues $T_{225}$ (orange), $Y_{190}$ (green), $F_{233}$ (red), and $K_{197}$ (purple) in the composite structure derived from crystals containing both Sfh1::Ptdlns and Sfh1::PtdCho unit cells (pdb 3B7Z).

(B) Plots monitoring the distances between the $\mathrm{C} \alpha$ atoms of residue pairs $\mathrm{Y}_{190}-\mathrm{T}_{225}$ and $\mathrm{K}_{197}-\mathrm{F}_{233}$ (as indicated in line charts). The last $8 \mathrm{~ns}$ of three independent simulations are shown in blue, orange, and green, respectively. Data obtained from Ptdlns-bound starting structures of Sec14, Sfh1, Sfh1 ${ }^{\mathrm{Y} 109 \mathrm{~A}}$, Sfh1 $1^{\mathrm{Y} 113 \mathrm{C}}$, and Sfh1 ${ }^{\mathrm{E} 126 \mathrm{~A}}$ are presented. Distances are given as a function of time (ns). (C) Distance fluctuations were calculated as relative SD of $\mathrm{C} \alpha$ atomic distances for residue pairs $\mathrm{Y}_{190}-\mathrm{T}_{225}$ (left) and $\mathrm{K}_{197}-\mathrm{F}_{233}$ (right). Averages and standard errors of fluctuations of three independent simulations are presented. Calculations from simulations using the Ptdlns-bound starting structures of Sec14, Sfh1, Sfh1 ${ }^{\mathrm{Y} 109 \mathrm{~A}}$, Sfh1 ${ }^{\mathrm{Y} 113 \mathrm{C}}$, and Sfh1 ${ }^{\mathrm{E} 126 \mathrm{~A}}$ are shown.

correlate Sfh1* with increased conformational fibrillations of the helical gate, suggesting that a primary mechanism for Sfh 1 * is a lowering of the energy barrier for initiating the closed-to-open transition necessary to facilitate $\mathrm{PL}$ exchange.

\section{DISCUSSION}

An understanding of the mechanisms by which Sec14-like PITPs integrate lipid metabolism with PIP signaling requires a detailed description of the mechanics by which these proteins execute heterotypic PL exchange. This information is of central importance given $\mathrm{PL}$ exchange lies at the heart of the mechanisms by which these proteins stimulate the activities of Ptdlns kinases in vivo (Schaaf et al., 2008; Bankaitis et al., 2010). Such questions assume larger significance given the broad conservation, across the Sec14 superfamily, of the structural elements that control ligand cycling into and from the protein interior, including cases of Sec14-like proteins whose dysfunction results in human disease (Ryan et al., 2007; Schaaf et al., 2008; Bankaitis et al., 2010). The available data suggest that the dynamics that govern the intramolecular motions (and biological activities) of Sec14-like proteins share general mechanistic principles. These dynamics are complex and involve coordination of chemical interactions on the protein surface (e.g., membrane binding), with those in the protein interior (e.g., with bound PL), and with the conformational transitions that gate access to the ligandbinding pocket. At present, we have no clear appreciation of the rules that govern the functional dynamics of Sec14-superfamily proteins. Herein, we describe how Sec14-like functions are activated in the natural pseudo-Sec14 (Sfh1). The directed evolution approach used in this study yielded gain-of-function Sfh1 derivatives. These
Sfh 1 * provide a novel prism through which the functional engineering of a Sec14-like protein can be observed. The concepts culled from these analyses are invisible to standard bioinformatic, crystallographic, and rational mutagenesis approaches.

We reach five major conclusions in this study: 1) Single residue substitutions are sufficient to endow Sfh1 with significantly enhanced abilities for promoting Sec14-dependent membrane trafficking events and for stimulation of Ptdlns 4-OH kinase activities in vivo. These substitutions show an informative allele specificity. 2) Missense substitutions that produce Sfh 1* involve residues conserved between Sec14 and Sfh1. Most of these residues cluster in an enigmatic hydrophilic microenvironment buried deep within the Sfh1 (and Sec14) hydrophobic pocket. 3) Both Ptdlns and PtdCho binding are required for Sfh $1{ }^{*}$-mediated potentiation of Ptdlns $4-\mathrm{OH}$ kinase activity in vivo, indicating that Sfh 1 * must execute heterotypic Ptdlns/PtdCho-exchange reactions for productive biological function. 4) Sfh $1^{*}$ are associated with increased cycling of PL into and out of the protein interior. 5) MD simulations project that Sfh1* facilitate a network of conformational transitions that enhance the dynamics of the helical substructure that gates the hydrophobic pocket. The collective results indicate Sfh 1 is a pseudo-Sec14 because it cannot efficiently propagate the intramolecular conformational transitions that support the robust heterotypic $\mathrm{PL}$ exchange required for potentiation of Ptdlns 4-OH kinase activity in vivo. These data also reveal mechanisms for how such transitions can be restored.

\section{Kinetic basis for mechanism of Sfh 1 *}

Because no intragenic synergies in potency of $\mathrm{SFH} 1^{*}$ phenotypes were observed (i.e., in combinatorial arrangements of the mutations studied in this report), we suggest a common activation mechanism is involved in the Sfh 1* examples highlighted here. Furthermore, the data point to a kinetic basis for the Sfh $1^{*}$ activation mechanism rather than a thermodynamic one. Our previous experiments demonstrated that Sfh1 is able to quantitatively load with Ptdlns or PtdCho under thermodynamically controlled (i.e., equilibrium) conditions (Schaaf et al., 2008). Yet we demonstrate enhanced PL cycling is a feature of the Sfh1* mechanism, and MD simulations indicate increased frequencies of helical gate opening (Figure 9). These independent analyses are both consistent with models in which PL exchange is kinetically favored in Sfh1* contexts. Finally, we calculated free bind-

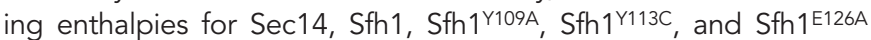
considering internal energies (bonds, angle, and torsion), van der Waals and electrostatic energies, nonpolar and electrostatic contributions to the solvation free energy, and contributions from the gas phase (see Supplemental Material and Methods). The relative protein-PL binding enthalpies calculated from MD simulations indicated the binding affinities of Sfh1, Sfh $1^{*}$, and Sec14 for Ptdlns were significantly stronger than for PtdCho (Supplemental Figure S8A). However, no significant Sfh $1{ }^{*}$-specific differences were discerned between binding enthalpies for the Ptdlns or PtdCho structures. 


\section{Functional connectivity between the hydrophilic microenvironment and the helical gate}

The hydrophilic patch residues whose alteration generates Sfh1* (i.e., $\mathrm{Y}_{109}, \mathrm{Q}_{111}, \mathrm{Y}_{113}$, and $\mathrm{E}_{126}$ ) reside in, or lie immediately adjacent to, the $B_{1} L B_{2}$-that is, one of the two substructures that define a critical conformational element termed the gating module (G-module; Ryan et al., 2007). As such, these directly influence the very elements that conduct propagation of conformational energy between the hydrophobic pocket floor and the helical substructure that gates access to the Sfh1 hydrophobic pocket. High-resolution structural data demonstrate the Sfh 1 * substitutions effect a remarkably subtle rearrangement of the hydrophilic microenvironment. However, both structural analyses and MD simulations indicate Sfh 1 * reconfigure interactions between side chains and ordered $\mathrm{H}_{2} \mathrm{O}$ molecules in this region. Analyses of the gain-of-function $\mathrm{Sfh} 1{ }^{*}$ substitutions demonstrate that the interaction between the $\mathrm{C}$-terminal region of strand $B_{2}$ (residues $E_{126}$ and $E_{127}$ ) with the $T_{4}$ turn in the string motif (residues $\mathrm{K}_{265}$ and $\mathrm{Y}_{266}$ ) is critical for the $\mathrm{Sfh}$ * phenotype. By contrast, strong interactions between the loop regions of the $B_{1} L_{2}\left(H_{114}\right.$, $\mathrm{V}_{116}$, and $\left.\mathrm{D}_{119}\right)$ and $\mathrm{A}_{11} \mathrm{LT}_{4}\left(\mathrm{Q}_{256}\right.$ and $\left.\mathrm{N}_{261}\right)$ substructures are incompatible with Sfh ${ }^{*}$.

\section{A role of buried water?}

The fact that Sfh 1 * substitutions involve Sfh1 residues that are conserved with Sec14 highlights the limitations of primary sequence alignments, with or without high-resolution structural information, in annotating protein function or in determining dynamic aspects of protein function. The $\mathrm{Sfh} 1$ * demonstrate that strong polar interside chain interactions (i.e., $E_{126}-Y_{124}$ and $E_{126}-Y_{109}$ ) are incompatible with the conformational dynamics required for Sec14-like functions in the Sfh1 context. The collective data suggest that interactions of these key side chains with water differ between Sfh1 and Sec14 and that internal waters play an important functional role in the regulation of $\mathrm{H}$-bond interactions within the hydrophobic pocket.

While the hydrophobic PL-binding cavity would seem an unlikely environment for active water flux, fatty acid-binding proteins exhibit significant internal water rearrangements coincident with ligand binding and release (Lücke et al., 2002; Modig et al., 2003). The available evidence indicates this is also the case for Sec14/ Sfh1. The internal water content of the hydrophobic pocket differs in the holo Sfh $1::$ Ptdlns and Sfh $1::$ PtdCho complexes as a direct result of the differential Ptdlns- and PtdCho-binding strategies, and water flux is suggested to contribute to the energetics that drive heterotypic PL exchange (Schaaf et al., 2008). The hydrophilic microenvironment plays a central role in this flux as it organizes a network of coordinated waters with the $Y_{109}, Q_{111}, Y_{124}$, and $E_{126}$ side chains playing primary roles (Schaaf et al., 2008; Figure 5B). Indeed, we find a surprisingly robust turnover of $\mathrm{H}_{2} \mathrm{O}$ in this region (as defined in the Supplemental Materials and Methods and Supplemental Figure S8B)_even after equilibrium conditions are reached (Supplemental Figure S8C). For instance, the average residence of $\mathrm{H}_{2} \mathrm{O}$ molecules in the proximity of core Sfh 1 * residues within a 2-ns time window is $<51 \%$ and $<60 \%$ for Sfh $1::$ PtdCho and Sfh $1::$ Ptdlns, respectively (Supplemental Figure S8C). These residence values are reduced to $25 \%$ for the complete course of the $14-n s$ MD simulation (unpublished data). Of note, the variability of simulated $\mathrm{H}_{2} \mathrm{O}$ turnover is both significantly and consistently increased in Sfh 1 * (Supplemental Figure S8, C and D). While a functional role for internal water is an attractive idea, it remains to be determined whether increased fluctuations in water turnover are essential features of Sfh 1 * activation mechanisms. This remains an open question for future address.

\section{Sec14 and Sfh1*-like, yet unlike}

Interestingly, Sfh1* do not precisely follow the Sec14 engineering blueprint for regulating their acquired Sec14-like conformational dynamics and activities. For example, decreased interactions of the $T_{4}$ turn with $\beta$-strand $B_{2}$ (i.e., $Y_{266} F$ and $Y_{266} D$ ) are incompatible with Sfh1*. Yet the corresponding Sec14 residue is $F_{264}$-not $Y_{264}$. The $\mathrm{F}_{264}$ residue seemingly disqualifies Sec14 from coupling the conformational transitions of these two conserved substructures through the specific $\mathrm{H}$-bonding mechanism utilized by Sfh 1 *. Use of an alternative conformational coupling mechanism for biologically sufficient rates of PL exchange reports a significant plasticity for how functional reactivation can be achieved in operationally inactive Sec14like proteins. Another line of evidence to that effect is provided by the $\mathrm{Q}_{204} \mathrm{R}$ Sfh1* substitution. Although not characterized in this report, Sfh 1 ${ }^{\mathrm{Q} 204 \mathrm{R}}$ involves a residue spatially removed from the physically clustered Sfh 1* residues upon which we focus here. The mechanism by which Sec14-like functions is resurrected in Sfh1 ${ }^{\text {Q204R }}$ is almost certainly distinct from the mechanisms that apply to Sfh 1 * substitutions that cluster in the hydrophilic microenvironment.

Finally, the results described in this report hold out the prospect that plasticity in conformational coupling can be used for the purpose of bypassing the normal requirements for specific structural elements in Sec14 (or a model Sec14-like protein). This capability might have interesting applications. A number of inherited human disease mutations in proteins of the Sec14 superfamily compromise the G-module, that is, the structural unit that transmits conformational information to the helical gate (Ryan et al., 2007). It might be feasible to "reactivate" such mutant proteins with small molecules that reprogram the conformational transitions upon which the biological activity of the Sec14-like protein depends.

\section{MATERIALS AND METHODS \\ Yeast strains}

Strains CTY182 (MATa ura3-52 lys2-801 $\Delta$ his3-200), CTY1-1A (MATa ura3-52 lys2-801 $\Delta$ his3-200 sec14-1 ts), CTY 100 (MATa ura3-52 lys2$801 \Delta$ his3-200 sec14-1ts sac1-26), CTY159 (MATa ura3-52 lys2-801 $\Delta$ his3-200 sec14-1ts kes1), CTY1079 (MATa ura3-52 lys2-801 $\Delta$ his3200 sec14-1ts spo144::HIS3), CTY1098 (MATa ura3-52 lys2-801 $\Delta$ his3-200 sec14-1ts kes1), CTY303 (MATa ura3-52 lys2-801 $\Delta$ his3$200 \Delta$ sec14, cki1::HIS3), CTY558 (MAT $\alpha$ ade2 ade3 leu2 $\Delta$ his3 ura3$52 \sec 14 \Delta 1:: H I S 3$ YEp[SEC14, LEU2, ADE3]), and BY4741 (MATa his $3 \Delta 1$ leu $2 \Delta 0$ met15 $\Delta 0$ ura3 $\Delta 0$ ) have been described previously (Cleves et al., 1991; Fang et al., 1996; Xie et al., 1998; Phillips et al., 1999; Li et al., 2000; EUROSCARF: http://web.uni-frankfurt.de/fb15/ mikro/euroscarf/index.html).

\section{Reagents}

Standard reagents were purchased from Sigma (St. Louis, MO) or Fisher (Pittsburgh, PA). All PLs-including nitroxide-labeled PtdCho species [1-acyl-2-(n-(4,4-dimethyloxazolidine- $N$-oxyl)stearoyl)-snglycero-3-phosphocholines; n-doxyl-PtdCho], with the doxyl label incorporated at $n=5,7,10,12$, or 16 of the sn-2 acyl chain-were purchased from Avanti (Alabaster, AL). Monoclonal Anti-V5 antibodies were obtained from Invitrogen (Carlsbad, CA); secondary antibodies were from Bio-Rad Laboratories (Hercules, CA).

\section{Media, genetic techniques, and PL-transfer assays}

Genetic methods, media, CPY pulse chase experiments, and PLtransfer assays have been described (Kearns et al., 1998; Phillips et al., 1999; Li et al., 2000; Yanagisawa et al., 2002; Schaaf et al., 2008). PIPs were extracted from yeast radiolabeled to steady state with $\left[{ }^{3} \mathrm{H}\right]$ myo-inositol and deacylated, and glycero-inositol 
phosphate derivatives were resolved and quantified by anionexchange chromatography (Guo et al., 1999; Rivas et al., 1999). Measurements of Ptdlns-4-P produced specifically by the Stt4 Ptdlns $4-\mathrm{OH}$ kinase were performed using $\sec 14-1^{\text {ts }} \operatorname{sac} 1 \Delta$ strains radiolabeled to steady state with $\left[{ }^{3} \mathrm{H}\right]$ myo-inositol as previously described (Phillips et al., 1999; Schaaf et al., 2008; lle et al., 2010).

\section{Protein expression, purification, and loading with PL}

His 8 -Sfh 1E126A was purified from Escherichia coli as described previously (Schaaf et al., 2006; Schaaf et al., 2008). To obtain Sfh1E126A::PtdCho complexes, 16:0/18:1 PtdCho dissolved in $\mathrm{CHCl}_{3}$ was dried under $\mathrm{N}_{2}$ in glass tubes. Lipid films were resuspended in $150 \mathrm{mM} \mathrm{NaCl}$ and $20 \mathrm{mM}$ Tris ( $\mathrm{pH} \mathrm{7.5)} \mathrm{and} \mathrm{sonicated} \mathrm{until} \mathrm{clear.}$ Liposomes were incubated with 40:1 M ratio of PtdCho::Sfh1E126A for $1 \mathrm{~h}$ at $37^{\circ} \mathrm{C}$. Complexes were repurified by binding and extensive washing on Talon cobalt-affinity resin (BD Biosciences, Clontech, Palo Alto, CA) followed by dialysis and gel filtration chromatography.

\section{Crystallization and structure determination}

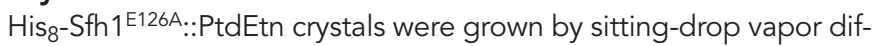
fusion at $22^{\circ} \mathrm{C}$ from solutions containing $1.5 \mu$ protein at $\sim 2.5 \mathrm{mg} / \mathrm{ml}$ (1.8 mg/ml) and $1.5 \mu$ crystallant: 25.5\% (wt/vol) PEG 4000, 11.9\% (vol/vol) glycerol, and $170 \mathrm{mM}$ sodium acetate and $85 \mathrm{mM}$ Tris, $\mathrm{pH}$ 7.5. Crystals were grown in cryoprotectant and flash cooled in liquid $\mathrm{N}_{2}$. Data to $1.8 \AA$ resolution were collected at $100 \mathrm{~K}$ at the South East Regional Collaborative Access Team beamline at Argonne National Laboratory (Argonne, IL) and processed and scaled with HKL2000 (Otwinowski, 1993). Initial phases were determined using the Sfh 1::PtdEtn structure (3B74) as a molecular replacement search model (Schaaf et al., 2008). Model building and refinement were performed using standard methods in Coot (Emsley and Cowtan, 2004) and CCP4 (1994, Oxon, UK), respectively. The final model contains one $\mathrm{His}_{8}$-Sfh $1^{\mathrm{E} 126 \mathrm{~A}}$ monomer (residues 4-309) and one bound PtdEtn in the asymmetric unit.

\section{Preparation of aqueous dispersion of $n$-doxyl-PtdCho and protein binding}

Multilamellar aqueous dispersions of n-doxyl-PtdChos were prepared in a phosphate $\mathrm{pH} 6.8$ buffer as previously described (Smirnova et al., 2007). Loading of Sfh1 proteins with n-doxyl-PtdCho was achieved by mixing a $130-\mu \mathrm{M}$ protein solution with twofold molar excess of $100 \%$ spin-labeled multilamellar liposomes and incubating at room temperature until no changes in EPR spectra were observed. In case of Sfh1, the EPR signals were also recorded $2 \mathrm{~d}$ after the mixing to verify the absence of any slow binding events. Effective order parameter, $S^{\text {eff }}$, was calculated as described in Schorn and Marsh (1997):

$$
S^{\text {eit }}=\frac{\left(A_{\|}-A_{\perp}\right) A_{0}}{\frac{1}{3}\left(A_{\|}+2 A_{\perp}\right) \Delta A}
$$

where $A_{\|}=A_{\text {out }}$, that is, the outer hyperfine splitting, and $A_{\perp}$ is calculated from $A_{i n}$, a half of the inner hyperfine splitting expressed in Gauss:

$$
\begin{aligned}
& A_{\perp}=\left(A_{\text {in }}+0.85\right) \text { for } S^{\text {app }}<0.45 \\
& A_{\perp}=A_{\text {in }}+1.32+1.86 \log \left(1-S^{\text {app }}\right) \text { for } S^{\text {app }}>0.45 \\
& S^{\text {app }}=\left(A_{\text {out }}-A_{\text {in }}\right) / \Delta A
\end{aligned}
$$

where $A_{0}$ is the isotropic nitrogen hyperfine coupling constant and $\Delta \mathrm{A}$ is the maximum extent of the axial nitrogen hyperfine anisotropy.
Although previously we have shown that the values of $\Delta \mathrm{A}$ and $\mathrm{A}_{0}$ can vary with position of the label along the sn-2 acyl chain for Sec14p-bound n-doxyl-PtdCho, we used the same values $A_{0}$ and $\Delta \mathrm{A}$ for all $n$-doxyl-PtdCho protein complexes. $A_{0}$ and $\Delta A$ were set to those measured for 5-doxyl-PtdCho in isopropanol (Smirnova et al., 2007).

\section{EPR spectroscopy and spectral analysis}

CW X-band (9.0-9.5 GHz) EPR spectra were acquired with a Century Series Varian E-109 (Varian Associates, Palo Alto, CA) EPR spectrometer and digitized to 2048 data points per spectrum. Spectrometer settings were as follows: Microwave power was $2 \mathrm{~mW}$, magnetic field modulation frequency was $100 \mathrm{kHz}$ with $<1 \mathrm{G}$ amplitude to avoid overmodulation, and magnetic field scan was $160 \mathrm{G}$. Sample temperature was $23.0 \pm 0.1^{\circ} \mathrm{C}$ controlled by a home-built variable temperature system (Alaouie and Smirnov, 2006).

\section{Supplemental material}

Supplemental material includes eight figures and three tables.

\section{ACKNOWLEDGMENTS}

This work was supported by grant GM44530 from the National Institutes of Health to V.A.B., and it supported the efforts of G.S., C.J.M., S.D.S., P. Y., K. T., and L.L.Y. G. S., M. D., E.M.W., and M.K.F.C. are supported by grants SCHA 1274/1-1 and Emmy Noether grant 1274/2-1 from the Deutsche Forschungsgemeinschaft, T. S. and M.C.Q. are supported by NSF-0843632, and E. O. is supported by Emory University Start-up Funds. We acknowledge the University of North Carolina Lineberger Comprehensive Cancer Center Genome Analysis and Nucleic Acids Core facilities. We also thank bwGRID, a member of the German D-Grid initiative, founded by the Ministry for Education and Research and the Ministry for Science, Research, and Arts Baden-Württemberg, for providing computational resources (www.bw-grid.de). Contact information for MD simulation data, marek-dynowski@web.de; EPR data, tismirno@ ncsu.edu; and structural determination, eric.ortlund@emory.edu.

\section{REFERENCES}

Alaouie AM, Smirnov Al (2006). Ultra-stable temperature control in EPR experiments: thermodynamics of gel-to-liquid phase transition in spinlabeled PL bilayers and bilayer perturbations by spin labels. J Mag Res 182, 229-238.

Balla T (2005). Inositol-lipid binding motifs: signal integrators through protein-lipid and protein-protein interactions. J Cell Sci 118, 2093-2104.

Bankaitis VA, Aitken JR, Cleves AE, Dowhan W (1990). An essential role for a phospholipid transfer protein in yeast Golgi function. Nature 347, 561-562

Bankaitis VA, Mousley CJ, Schaaf G (2010). The Sec14-superfamily and mechanisms for crosstalk between lipid metabolism and lipid signaling. Trends Biochem Sci 35, 150-160.

Benomar M et al. (2002). Clinical comparison between AVED patients with 744 del A mutation and Friedreich ataxia with GAA expansion in 15 Moroccan families. J Neurol Sci 198, 25-29.

Bomar JM et al. (2003). Mutations in a novel gene encoding a CRAL-TRIO domain cause human Cayman ataxia and ataxia/dystonia in the jittery mouse. Nature Genetics 35, 264-269.

Cleves AE, McGee TP, Whitters EA, Champion KM, Aitken JR, Dowhan W, Goebl M, Bankaitis VA (1991). Mutations in the CDP-choline pathway for phospholipid biosynthesis bypass the requirement for an essential phospholipid transfer protein. Cell 64, 789-800.

Cleves AE, Novick PJ, Bankaitis VA (1989). Mutations in the SAC1 gene suppress defects in yeast Golgi and yeast actin function. J Cell Biol 109 2939-2950.

D'Angelo I, Welti S, Bonneau F, Scheffzek K (2006). A novel bipartite phospholipid-binding module in the neurofibromatosis type 1 protein. EMBO Rep 7, 174-179. 
Emsley P, Cowtan K (2004). Coot, model-building tools for molecular graphics. Acta Crystallographica D60, 2126-2132.

Fang M, Kearns BG, Gedvilaite A, Kagiwada S, Kearns M, Fung MKY, Bankaitis VA (1996). Kes1 shares homology with human oxysterol binding protein and participates in a novel regulatory pathway for yeast Golgi-derived transport vesicle biogenesis. EMBO J 15, 6447-6459.

Foti M, Audhya A, Emr SD (2001). Sac1 lipid phosphatase and Stt4 phosphatidylinositol-4-kinase regulate a pool of phosphatidylinositol 4-phosphate that functions in control of the actin cytoskeleton and vacuole morphology. Mol Biol Cell 12, 2396-2411.

Fruman DA, Meyers RE, Cantley LC (1998). Phosphoinositide kinases. Annu Rev Biochem 67, 481-507.

Guo S, Stolz LE, Lemrow SM, York JD (1999). SAC1-like domains of yeast SAC1, INP52, and INP53 and of human synaptojanin encode polyphosphoinositide phosphatases. J Biol Chem 274, 12990-12995.

lle KE et al. (2010). The zebrafish class 1 phosphatidylinositol transfer protein family, PITP $\beta$ isoforms and double cone cell outer segment integrity in retina. Traffic 11, 1151-1167.

Ile KE, Schaaf G, Bankaitis VA (2006). Phosphatidylinositol transfer proteins and cellular nanoreactors for lipid signaling. Nature Chem Biol 2, 576-583.

Kearns MA et al. (1998). Novel developmentally regulated phosphoinositide binding proteins from soybean whose expression bypasses the requirement for an essential phosphatidylinositol transfer protein in yeast. EMBO J 17, 4004-4017.

Lemmon MA (2008). Membrane recognition by phospholipid-binding domains. Nat Rev Mol Cell Biol 9, 99-111.

Li X, Routt S, Xie Z, Cui X, Fang M, Kearns MA, Bard M, Kirsch D, Bankaitis VA (2000). Identification of a novel family of nonclassical yeast PITPs whose function modulates activation of phospholipase D and Sec14p-independent cell growth. Mol Biol Cell 11, 1989-2005.

Lücke C, Huang S, Rademacher M, Rüterjans H (2002). New insights into intracellular lipid binding proteins: the role of buried water. Protein Sci $11,2382-2392$.

Majerus PW (1997). Inositol phosphatases and kinases in cell signaling. FASEB J 11, A1297.

McLaughlin S, Murray D (2005). Plasma membrane phosphoinositide organization by protein electrostatics. Nature 438, 605-611.

Meier R, Tomizaki T, Schulze-Briese C, Baumann U, Stocker A (2003). The molecular basis of vitamin $E$ retention: structure of human tocopherol transfer protein. J Mol Biol 331, 725-734.

Modig K, Rademacher M, Lücke C, Halle B (2003). Water dynamics in the large cavity of three lipid-binding proteins monitored by ${ }^{17} \mathrm{O}$ magnetic relaxation dispersion. J Mol Biol 332, 965-977.

Nemoto Y, Kearns BG, Wenk MR, Chen H, Mori K, Alb JG Jr, De Camilli P, Bankaitis VA (2000). Functional characterization of a mammalian Sac1 and mutants exhibiting substrate specific defects in PIP phosphatase activity. J Biol Chem 275, 14446-14456.

Otwinowski Z (1993). Data collection and processing. In Proceedings of the CCP4 Study Weekend, Sawyer L, Isaacs N, and Bailey S, eds., Daresbury, UK: SERC Danesbury Laboratory, 56-62.
Ouachi K, Arita M, Kayden H, Faycal H, Hamida MB, Sokol R, Arai H, Inoue $\mathrm{K}$, Mandel J-L, Koenig M (1995). Ataxia with vitamin E deficiency is caused by mutations in the $\alpha$-tocopherol transfer protein. Nature Genetics 9, 141-145.

Phillips SE et al. (1999). Yeast Sec14p deficient in phosphatidylinositol transfer activity is functional in vivo. Mol Cell 4, 187-197.

Rivas MP, Kearns BG, Xie Z, Guo S, Sekar MC, Hosaka K, Kagiwada S, York JD, Bankaitis VA (1999). pleiotropic alterations in lipid metabolism in yeast sac1 mutants: relationship to "bypass Sec14p" and inositol auxotrophy. Mol Biol Cell 10, 2235-2250.

Routt SM, Ryan MM, Tyeryar K, Rizzieri K, Roumanie O, Brennwald PJ، Bankaitis VA (2005). Nonclassical PITPs activate phospholipase D via an $\mathrm{Stt} 4 \mathrm{p}$-dependent pathway and modulate function of late stages of the secretory pathway in vegetative yeast cells. Traffic 6, 1157-1172.

Ryan MM, Temple BRS, Phillips SE, Bankaitis VA (2007). Conformational dynamics of the major yeast phosphatidylinositol transfer protein Sec14: insights into the mechanisms of PL exchange and diseases of Sec14-like protein deficiencies. Mol Biol Cell 18, 1928-1942.

Schaaf G, Betts L, Garrett TA, Raetz CRH, Bankaitis VA (2006). Crystallization and preliminary $\mathrm{X}$-ray diffraction analysis of phospholipid-bound Sfh1p: a member of the Saccharomyces cerevisiae Sec14p-like phosphatidylinositol transfer protein family. Acta Crystallographica F 62, 1156-1160.

Schaaf G, Ortlund E, Tyeryar K, Mousley C, lle K, Woolls M, Garrett T, Raetz $\mathrm{CRH}$, Redinbo M, Bankaitis VA (2008). The functional anatomy of phospholipid binding and regulation of phosphoinositide homeostasis by proteins of the Sec14-superfamily. Molecular Cell 29, 191-206.

Schorn K, Marsh D (1997). Extracting order parameters from powder EPR lineshapes for spin-labelled lipids in membranes. Spectrochimica Acta A 53, 2235-2240.

Sha B, Phillips SE, Bankaitis VA, Luo M (1998). Crystal structure of the Saccharomyces cerevisiae phosphatidylinositol transfer protein Sec14p. Nature 391, 506-510.

Smirnova T, Chadwick TG, MacArthur R, Poluekov O, Song L, Ryan M, Schaaf G, Bankaitis VA (2006). The chemistry of PL binding by the Saccharomyces cerevisiae phosphatidylinositol transfer protein Sec14 as determined by electron paramagnetic resonance spectroscopy. J Biol Chem 281, 34897-34908.

Smirnova T, Chadwick TG, van Tol J, Ozarowski A, Poluektov O, Schaaf G, Ryan MM, Bankaitis VA (2007). Local polarity and hydrogen bonding inside the Sec14 PL-binding cavity: high-field multifrequency studies. Biophys J 92, 3686-3695.

Strahl T, Thorner J (2007). Synthesis and function of membrane phosphoinositides in budding yeast, Saccharomyces cerevisiae. Biochim Biophys Acta 1771, 353-404.

Xie Z, Fang M, Rivas MP, Faulkner A, Sternweis PC, Engebrecht J, Bankaitis VA (1998). Phospholipase $D$ activity is required for suppression of yeast phosphatidylinositol transfer protein defects. Proc Natl Acad Sci USA 95, 12346-12351.

Yanagisawa L, Marchena J, Xie Z, Li X, Poon PP, Singer R, Johnston G, Randazzo PA, Bankaitis VA (2002). Activity of specific lipid-regulated ARFGAPs is required for Sec14p-dependent Golgi secretory function in yeast. Mol Biol Cell 13, 2193-2206. 